\title{
EQUIVARIANT MINIMAL MODELS
}

BY

\author{
GEORGIA V. TRIANTAFILLOU ${ }^{1}$
}

\begin{abstract}
Let $G$ be a finite group. We give an algebraicization of rational $G$-homotopy theory analogous to Sullivan's theory of minimal models in ordinary homotopy theory.
\end{abstract}

1. Introduction. We consider simplicial complexes $X$ on which a finite group $G$ acts simplicially. We assume that $X^{g}=\{x \in X \mid g x=x\}$ is a simplicial complex for each $g \in G$; this can always be arranged by repeated triangulation. We assume once and for all that all $G$-spaces in sight are $G$-simple in the sense that the fixed point spaces $X^{H}$ are simple for all subgroups $H$ of $G$. We also assume that the rational cohomology of each $X^{H}$ is of finite type.

Definition 1.1. A $G$-map $f: X \rightarrow Y$ is a rational $G$-homotopy equivalence if each $f^{H}: X^{H} \rightarrow Y^{H}$ induces an isomorphism on rational cohomology.

Definition 1.2. Two $G$-spaces $X$ and $Y$ have the same rational $G$-homotopy type if there is a chain of rational $G$-homotopy equivalences connecting them.

These definitions are motivated by the following equivariant version of the Whitehead theorem [1]:

TheOREM 1.3 (G. BREDON). An equivariant map $f: X \rightarrow Y$ between two $G$-complexes is an equivariant homotopy equivalence iff $\left(f^{H}\right)_{*}: \pi_{*}\left(X^{H}\right) \rightarrow \pi_{*}\left(Y^{H}\right)$ is an isomorphism for each subgroup $H \subseteq G$.

By using the same equivariant obstruction theory which leads to this result, we constructed in [5] an equivariant Postnikov decomposition of a $G$-complex $X$, i.e., a decomposition of $X$ in a sequence of $G$-fibrations

$$
\{p t\} \leftarrow X_{1} \leftarrow \cdots \leftarrow X_{n-1} \leftarrow X_{n} \leftarrow \cdots,
$$

the fibers $F_{n}$ of which have the property that $F_{n}^{H}$ is an Eilenberg-Mac Lane space of dimension $n$ for each $H \subseteq G$ (we can speak of a $G$-action on the fiber since $X$ has at least one fixed point). We shall review this material in $\$ 2$. Our goal is to construct an equivariant minimal model for $X$ which is the algebraic analogue of the rationalization (localization at 0 ) of the equivariant Postnikov decomposition of $X$.

Received by the editors June 12, 1980 and, in revised form, July 15, 1980 and June 24, 1981 .

1980 Mathematics Subject Classification. Primary 55P10, 57S17; Secondary 55S45.

Key words and phrases. G-complex, rationalization, differential algebra, minimal model, equivariant Postnikov system.

'Supported in part by an NSF grant through the Institute for Advanced Study, Princeton, N. J. 
Sullivan's minimal model of a simplicial complex $X$ is a differential graded Q-algebra (abbreviated DGA), $\mathfrak{N}_{X}$ which is free and has decomposable differential and which maps by a cohomology isomorphism into the de Rham algebra $\mathcal{E}_{X}$ of differential forms on $X$. In the case of a $G$-complex, we consider not only the DGA $\mathcal{E}_{X}$ with the induced $G$-action on it, but also the de Rham algebras $\mathcal{E}_{X^{H}}$ of all the fixed point sets $X^{H}$ and the canonical maps relating them. This means that we consider a functor $\underline{\mathscr{E}}_{X}$ of a particular sort.

Let $\vartheta_{G}$ be the category of canonical orbits. Its objects are the orbits $G / H$ and its morphisms are the $G$-maps between them. We define a (contravariant) rational coefficient system for $G$ to be a contravariant functor from $\theta_{G}$ to the category of rational vector spaces. Let $\mathrm{Vec}_{G}$ denote the category of rational coefficient systems and let $\mathrm{Vec}_{G}^{*}$ denote the dual category of covariant functors from $\theta_{G}$ to rational vector spaces. An understanding of the injective objects of the abelian category $\mathrm{Vec}_{G}^{*}$ turns out to be central to our work, and we analyze the projective objects of $\mathrm{Vec}_{G}$ in $\S 3$.

Define a system of DGA's to be a covariant functor $\mathfrak{A}$ from $\theta_{G}$ to the category of (simple) DGA's which is injective when regarded as (graded) object of $\mathrm{Vec}_{G}^{*}$ by neglect of structure.

The functor $\underline{\mathcal{E}}_{X}$ above is a system of DGA's. We shall prove this in $\S 4$.

In $\S 5$ we shall specify a notion of minimal system of DGA's. This will allow us to prove the following result, which is the technical heart of our work, in $§ 7$ :

THEOREM 1.4. For each system of DGA's $\mathfrak{A}$, there is a minimal system of $D G A$ 's $\mathfrak{M}$ and a map of systems of DGA's $\rho: \mathfrak{M} \rightarrow \mathfrak{A}$ which induces an isomorphism on cohomolgy (at each $G / H$ ).

We call $\mathfrak{M}$ the minimal model of $\mathfrak{A}$. It is unique up to isomorphism. We then define the minimal model $\mathfrak{M}_{X}$ of $X$ to be the minimal model of $\underline{\mathcal{E}}_{X}$. With this definitional apparatus, we prove the following theorem in $\S 6$ :

THEOREM 1.5. The correspondence $X \mapsto \mathfrak{M}_{X}$ induces a bijection between equivariant rational homotopy types of G-spaces on the one hand and isomorphism classes of minimal systems of DGA's on the other.

Say that a $G$-complex $Y$ is rational if each homotopy group $\pi_{n}\left(Y^{H}\right)$ is a rational vector space. We shall define the notion of homotopy between morphisms of systems of DGA's in $\S 4$ and we shall prove the following theorem in $\S 6$ :

THEOREM 1.6. If $X$ is a G-complex and $Y$ a rational G-complex, there is a bijection

$$
[X, Y]_{G} \cong\left[\mathfrak{M}_{Y}, \mathfrak{M}_{X}\right] \text {. }
$$

This algebraicizes rational $G$-homotopy classes of $G$-maps.

Of course, this work presupposes the appropriate notion of cohomology which is that introduced by Bredon [1] and recalled in $\$ 2$. In a sense to be made precise, the minimal model $\mathfrak{M}_{X}$ computes the rational Bredon cohomology of $X$.

REMARK ON NOTATION. Throughout this paper $G$ stands for a fixed finite group, $H \subseteq G$ means $H$ is a subgroup of $G$ and $H \subset G$ means $H$ is a proper subgroup of $G$. 
I would like to thank Professors Dan Burghelea and J. Peter May for carefully reading the original manuscript and for useful suggestions.

2. The equivariant Postnikov decomposition and its rationalization. In this section we give a summary of Bredon's equivariant cohomology and obstruction theory [1] and of our construction in [5] of the equivariant Postnikov decomposition and its rationalization.

We first describe the morphisms in the category of canonical orbits $\theta_{G}$. Any morphism, i.e., any equivariant map $G / H \rightarrow G / K$, is of the form $g H \stackrel{\hat{a}}{\mapsto} g a H$ for some $a \in G$ with the property $a^{-1} H a \subseteq K$. Moreover, $\hat{a}=\hat{b}$ iff $a b^{-1} \in K$. It can be also easily checked that

$$
E(G / H, G / H) \cong N(H) / H \cong(G / H)^{H}
$$

where the first set is the set of $G$-maps $G / H \rightarrow G / H$ and $N(H)$ is the normalizer of $H$. The bijections are given by $N(H) \ni a \mapsto a H \in(G / H)^{H}$ and $a \mapsto \hat{a} \in$ $E(G / H, G / H), \hat{a}(g H)=g a H$. In general $E(G / K, G / H) \cong(G / H)^{K}$.

$A$ coefficient system for $G$ is a contravariant functor from $\theta_{G}$ to the category of abelian groups.

EXAMPLES. (1) If $A$ is a Z $[G]$-module, define a coefficient system $\underline{A}$ by $\underline{A}(G / H)=$ $A^{H}$, with $\underline{A}(\hat{a}): A^{K} \rightarrow A^{H}$ specified by $\underline{A}(\hat{a})(x)=a x$. Note that the multiplication $a$ : $A \rightarrow A$ maps $A^{K}$ into $A^{H}$ since $x \in A^{K}$ implies $h a x=a k a^{-1} a x=a k x=a x$, where $h \in H$ and $k \in K$.

(2) If $X$ is a $G$-complex, define coefficient systems $\underline{\pi}_{*}(X), \underline{H}_{*}(X)$ and $\underline{C}_{*}(X)$ by

$$
\underline{\pi}_{*}(X)(G / H)=\pi_{*}\left(X^{H}\right), \quad \underline{H}_{*}(X)(G / H)=H_{*}\left(X^{H} ; \mathbf{Z}\right)
$$

and

$$
\underline{C}_{*}(X)(G / H)=C_{*}\left(X^{H}\right)
$$

on objects, where $C_{n}(X)=H_{n}\left(X^{(n)}, X^{(n-1)} ; \mathbf{Z}\right)$. A morphism $\hat{a}: G / H \rightarrow G / K$ induces a map $a: X^{K} \rightarrow X^{H}$ and therefore a map on homotopy, homology and cell complex.

Let $M$ be any coefficient system for $G$. Since the category of such systems is abelian (see [4, p. 258]), the sets $\operatorname{Hom}\left(\underline{C}_{n}(X), M\right)$ are abelian groups. Moreover, they form a cochain complex with coboundary operator induced by the boundary operator of $\underline{C}_{*}(X)$. The equivariant cohomology of the $G$-complex $X$ is defined by

$$
H_{G}^{n}(X ; M)=H^{n}\left(\operatorname{Hom}\left(\underline{C}_{*}(X), M\right)\right) .
$$

The relation between $H_{G}^{n}(X ; M)$ and $\underline{H}_{*}(X)$ is given by a spectral sequence. The category of coefficient systems for $G$ has enough injectives, hence we can construct an injective resolution $M^{(*)}$ of $M$. The coefficient system $\underline{C}_{*}(X)$ is a projective object in this category. We consider the double complex $\operatorname{Hom}\left(\underline{C}_{*}(X), M^{(*)}\right)$. Standard homological algebra applied to this complex yields a spectral sequence

$$
E_{2}^{s, t}=\operatorname{Ext}^{s}\left(\underline{H}_{t}(X), M\right) \Rightarrow H_{G}^{s+t}(X ; M) \text {. }
$$


The relative equivariant cohomology $H_{G}^{n}(X, Y ; M)$ is also defined and there is a long exact sequence

$$
\cdots \rightarrow H_{G}^{n}(X, Y ; M) \rightarrow H_{G}^{n}(X ; M) \rightarrow H_{G}^{n}(Y ; M) \rightarrow H_{G}^{n+1}(X, Y ; M) \rightarrow \cdots .
$$

This is the right cohomology theory in which to express obstructions for extending or lifting $G$-maps.

(2.3) Equivariant obstruction theory. Let $Y$ be a $G$-subcomplex of $X$ and $\varphi$ : $X^{(n)} \cup Y \rightarrow Z$ be a $G$-map. Let $\sigma$ be an $(n+1)$-simplex of $X$ and $G_{\sigma} \subseteq G$ its isotropy group. It is clear that $\varphi\left(S^{n}\right) \subseteq Z^{G_{\sigma}}$, where $S^{n}$ is the boundary of $\sigma$, i.e., $\varphi \mid S^{n}$ defines an element in $\pi_{n}\left(Z^{G_{\sigma}}\right)$. Hence $\varphi$ defines a natural transformation $c_{\varphi} \in \operatorname{Hom}\left(\underline{C}_{n+1}(X, Y) ; \underline{\pi}_{n}(Z)\right)$. This is a cocycle and defines a cohomology class

$$
\left[c_{\varphi}\right] \in H_{G}^{n+1}\left(X, Y ; \underline{\pi}_{n}(Z)\right),
$$

which is the obstruction to extending $\varphi \mid X^{(n-1)} \cup Y$ to a $G$-map $X^{(n+1)} \cup Y \rightarrow Z$.

Let $M$ be a coefficient system for $G$. An Eilenberg-Mac Lane G-complex of type $(M, n)$ is a $G$-complex $K$ with the property $\underline{\pi}_{n}(K)=M$ and $\underline{\pi}_{m}(K)=0$ for $m \neq n$. In other words, $K^{H}$ is an Eilenberg-Mac Lane space of dimension $n$ for every $H \subseteq G$. Such $G$-complexes exist and fulfill the relation

$$
[X, K]_{G} \cong H_{G}^{n}(X ; M) \text {. }
$$

We may indicate one way an Eilenberg-Mac Lane $G$-complex can be constructed because of the analogy between this construction and an algebraic construction in $\S 5$. First, observe that there are enough projectives in the category of coefficient systems. One can construct a projective coefficient system as follows. Let $T$ be a $G$-set and let $\underline{F}(T)$ be defined by $\underline{F}(T)(G / H)=F\left(T^{H}\right)$, where $F\left(T^{H}\right)$ is the free abelian group generated by $T^{H}$ (note that $F\left(T^{H}\right) \neq F(T)^{H}$ ). For instance, $C_{n}(X)$ is a projective coefficient system of this form; here $T$ consists of the $n$-cells of $X$. In order to construct an Eilenberg-Mac Lane $G$-complex $K(M, n)$, first consider a projective resolution of $M$

$$
\cdots \rightarrow \underline{F}\left(T_{1}\right) \stackrel{f_{1}}{\rightarrow} \underline{F}\left(T_{0}\right) \rightarrow M \rightarrow 0 .
$$

Let $S^{n} T_{0}^{+}$be the one point union of $n$-spheres, one for each element of $T_{0}$, and let $C S^{n} T_{1}^{+}$be the one point union of $(n+1)$-cells, one for each element of $T_{1}$. In analogy with the construction of ordinary Eilenberg-Mac Lane complexes, the $G$-complex $S^{n} T_{0}^{+} \cup_{f_{1}} C S^{n} T_{1}^{+}$has the right homotopy groups in dimension $n$. The higher homotopy groups are killed by attaching cells similarly.

An equivariant map $p: E \rightarrow X$ is a $G$-fibration if it has the equivariant homotopy lifting property. Let $e_{0} \in E^{G}$ be a base point and put $x_{0}=p\left(e_{0}\right)$. The $G$-space $F=p^{-1}\left(x_{0}\right)$ is called the fiber of $p$. Let $Y$ be a $G$-complex, $f: X \rightarrow Y$ a $G$-map and $\tilde{f}$ : $Y^{(n)} \rightarrow E$ an equivariant lifting of $f \mid Y^{(n)}$, i.e., $\rho \circ \tilde{f}=f \mid Y^{(n)}$. There is a cohomology class

$$
\left[c_{(f, \tilde{f})}\right] \in H_{G}^{n+1}\left(Y ; \underline{\pi}_{n}(F)\right)
$$

which vanishes iff the lifting $\tilde{f} \mid Y^{(n-1)}$ can be extended equivariantly to a lifting $Y^{(n+1)} \rightarrow E$. Let $H$ be a $G$-homotopy between two such liftings $\tilde{f} \mid Y^{(n)}$ and $\tilde{f}^{\prime} \mid Y^{(n)}$. 
The obstruction to extending $H \mid Y^{(n-1)}$ to a $G$-homotopy $Y^{(n+1)} \times I \rightarrow E$ lies in $H_{G}^{n}\left(Y ; \pi_{n}(F)\right)$.

We refer to [1] for details of the material so far.

(2.4) A classification theorem follows easily from the obstruction theory above. Let $p: E \rightarrow X$ be a $G$-fibration, the fiber of which is an Eilenberg-Mac Lane $G$-complex $K(M, n)$. Let $f: Y \rightarrow X$ be a $G$-map and $\tilde{f}: Y \rightarrow E$ a fixed equivariant lifting of $f$. Then there is a bijection between the set of equivariant homotopy classes of equivariant liftings of $f$ on the one hand and $H_{G}^{n}(Y ; M)$ on the other. A class [ $\left.\tilde{f}^{\prime}\right]$ corresponds to the first obstruction to the existence of a $G$-homotopy between $\tilde{f}$ and $\tilde{f}^{\prime}$.

(2.5) The equivariant Postnikov decomposition of a $G$-space $X$ is a chain of $G$-spaces and $G$-maps such that the first diagram below commutes and the second is the ordinary Postnikov decomposition of $X^{H}$ for every $H \subseteq G$. We insist that $p_{n}$ : $X_{n} \rightarrow X_{n-1}$ be a $G$-fibration. Then the fiber is obviously an Eilenberg-Mac Lane $G$-complex $K\left(\underline{\pi}_{n}(X), n\right)$.
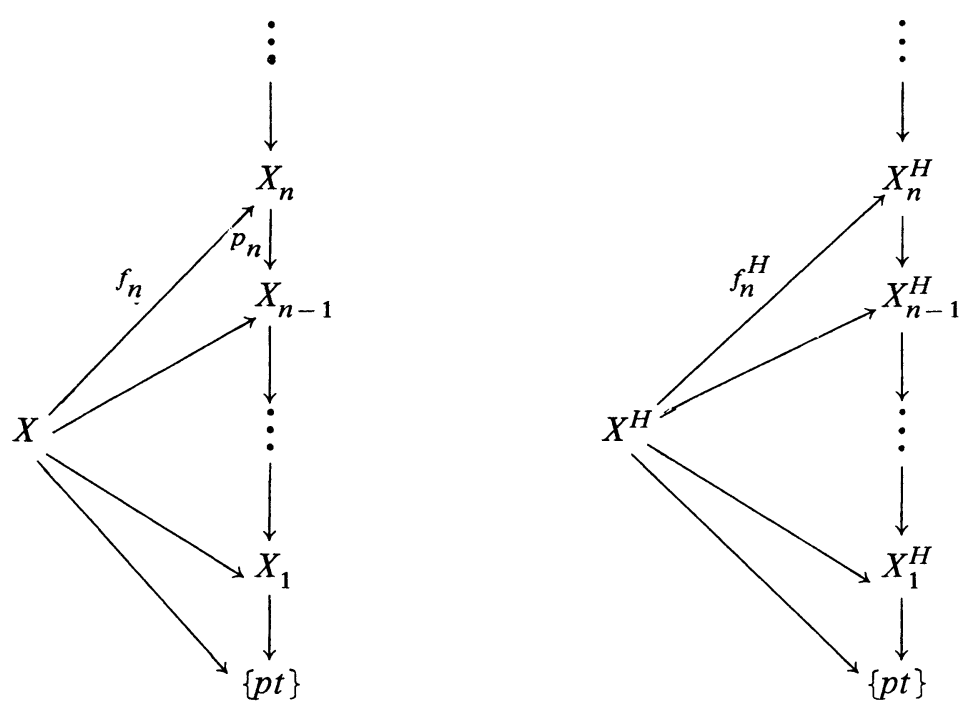

A $G$-fibration, the fiber of which is an Eilenberg-Mac Lane $G$-complex $K(M, n)$, is called a principal $G$-fibration. $G$-equivalence classes of principal $G$-fibrations over $X_{n-1}$ are classified by $H_{G}^{n+1}\left(X_{n-1} ; M\right) \cong\left[X_{n-1}, K(M, n+1)\right]_{G}$. Given a cohomology class in $H_{G}^{n+1}\left(X_{n-1}, M\right)$, i.e., a $G$-map $f: X_{n-1} \rightarrow K(M, n+1)$, we can construct a $G$-fibration over $X_{n-1}$ by considering the induced fibration by $f$ of the path fibration

$$
\Omega K(M, n+1) \rightarrow P K(M, n+1) \rightarrow K(M, n+1) .
$$

So the $G$-fibration $p_{n}: X_{n} \rightarrow X_{n-1}$ is characterized by a cohomology class $k^{n+1} \in$ $H_{G}^{n+1}\left(X_{n-1} ; \pi_{n}(X)\right)$, the $(n+1)$ st equivariant $k$-invariant of $X$.

The equivariant $k$-invariants of a $G$-space $X$ are determined by $X$ in the following way: By using the spectral sequence above we can prove

$$
H_{G}^{n+1}\left(X_{n-1}, X ; \underline{\pi}_{n}(X)\right) \cong \operatorname{Hom}\left(\underline{\pi}_{n}(X), \underline{\pi}_{n}(X)\right) .
$$


Then the cohomology class $\iota_{n}$ corresponding to id $\in \operatorname{Hom}\left(\underline{\pi}_{n}(X), \underline{\pi}_{n}(X)\right)$ is mapped by

$$
H_{G}^{n+1}\left(X_{n-1}, X ; \underline{\pi}_{n}(X)\right) \rightarrow H_{G}^{n+1}\left(X_{n-1} ; \underline{\pi}_{n}(X)\right)
$$

to $k^{n+1}$. The $G$-Postnikov decomposition is unique up to $G$-homotopy equivalence. Moreover, by passage to inverse limits, the $G$-Postnikov decomposition determines a $G$-space whose semisimplicial resolution (after barycentric subdivision) is a $G$-complex $G$-homotopy equivalent to $X$.

(2.6) Equivariant rationalization. A $G$-space $X$ is said to be rational ( $a \mathbf{Q}$ - $G$-space) if the homotopy groups $\pi_{i}\left(X^{H}\right)$ are Q-vector spaces for each $H \subseteq G$. For each $G$-space $X$ there exist a $\mathbf{Q}-G$-space $X_{0}$ and a $G$-map $f: X \rightarrow X_{0}$ such that the following equivalent statements hold:

(1) $f^{*}: H_{G}^{*}(X ; M \otimes \mathbf{Q}) \rightarrow H_{G}^{*}\left(X_{0} ; M \otimes \mathbf{Q}\right)$ is an isomorphism for all coefficient systems $M$;

(2) $f_{*}: \underline{H}_{*}(X ; \mathbf{Q}) \rightarrow \underline{H}_{*}\left(X_{0}, \mathbf{Q}\right) \cong \underline{H}_{*}\left(X_{0}, \mathbf{Z}\right)$ is an isomorphism;

(3) $f_{*} \otimes$ id: $\underline{\pi}_{*}(X) \otimes \mathbf{Q} \rightarrow \underline{\pi}_{*}\left(X_{0}\right) \otimes \mathbf{Q} \cong \underline{\pi}_{*}\left(X_{0}\right)$ is an isomorphism;

(4) the map $f$ has the universal lifting property, i.e., every $G$-map $g: X \rightarrow Y$, where $Y$ is a Q- $G$-space, factors through $X_{0}$, uniquely up to $G$-homotopy,

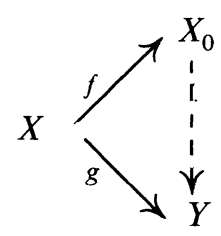

The existence of a $G$-rationalization of $X$ is proved by inductively constructing $G$-rationalizations $\varphi_{n}$ of the $G$-spaces $X_{n}$ of the $G$-Postnikov decomposition. We first localize the Eilenberg-Mac Lane $G$-complexes by constructing

$$
K\left(\underline{\pi}_{n}(X), n+1\right) \stackrel{i}{\rightarrow} K\left(\underline{\pi}_{n}(X) \otimes \mathbf{Q}, n+1\right)
$$

and then consider the "localized" $k$-invariants $k_{0}^{n+1}$ :

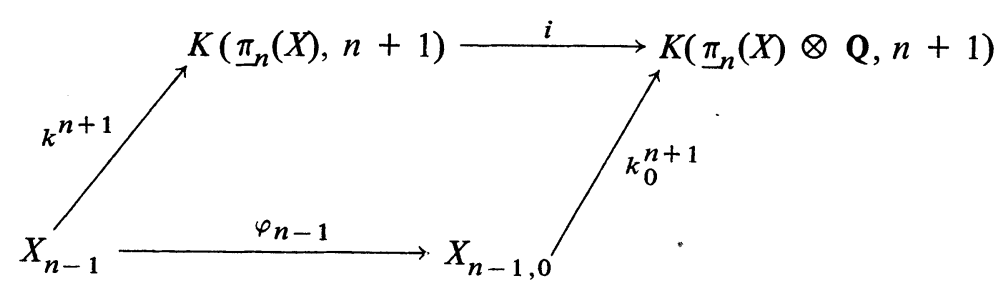

We can say, equivalently to Definition 1.2 , that the $G$-rational homotopy type of a $G$-space $X$ corresponds bijectively to the $G$-homotopy type of its rationalization $X_{0}$. 
3. Projective rational coefficient systems. In this section we shall study the projective (contravariant) rational coefficient systems and give a complete description of projective covers.

The category $\mathrm{Vec}_{G}$ of rational coefficient systems is abelian and a morphism $f$ : $M \rightarrow N$ is an epimorphism (respectively monomorphism) iff $f(G / H): M(G / H) \rightarrow$ $N(G / H)$ is an epimorphism (respectively monomorphism) of vector spaces. The examples of coefficient systems given in $\$ 2$ can obviously be tensored over $\mathbf{Q}$ to give rational coefficient systems. As before, given a $G$-set $T$, we can define a projective rational coefficient system $\underline{\mathbf{Q}}(T)$ by $\underline{\mathbf{Q}}(T)(G / H)=\mathbf{Q}\left(T^{H}\right)$, where $\mathbf{Q}\left(T^{H}\right)$ is the vector space over $\mathbf{Q}$ with basis $T^{H}$. We shall describe all projectives in $\mathrm{Vec}_{G}$.

We first make some easily checked elementary remarks. The set $G / H$ can be written as disjoint union

$$
G / H=(G / H)^{H} \cup(G / H)^{H_{1}} \cup \cdots \cup(G / H)^{H_{m}},
$$

where $H=H_{0}, H_{1}, \ldots, H_{m}$ are the distinct subgroups of $G$ conjugate to $H$. The sets $(G / H)^{H_{i}}$ are isomorphic, and $N(H) / H$ acts freely and transitively on each from the right by $(g H) a=(g a) H$. The action of $G$ on $G / H$ by left multiplication permutes the copies $(G / H)^{H_{i}}$, and $(G / H)^{K}$ contains those copies $(G / H)^{H_{i}}$ for which $K \subseteq H_{i}$. Thus $N(H) / H$ acts on $(G / H)^{K}$ from the right. The vector space $\mathbf{Q}\left((G / H)^{K}\right)$ is a free right $\mathbf{Q}(N(H) / H)$-module, a basis of which is any set of elements of $(G / H)^{K}$ such that exactly one element belongs to each copy $(G / H)^{H_{i}} \subseteq(G / H)^{K}$. Therefore, if $V_{H}$ is a left $\mathbf{Q}(N(H) / H)$-module, the tensor product $\left(\mathbf{Q}(G / H)^{K}\right) \otimes_{\mathbf{Q}(N(H) / H)} V_{H}$ is equal to a direct sum of copies of $V_{H}$. Of course, $V_{H}$ is necessarily projective since the group ring $\mathbf{Q}(N(H) / H)$ is semisimple.

Definition 3.1. Define a rational coefficient system $\underline{V}_{H}$ by

$$
\underline{V}_{H}(G / K)=\mathbf{Q}\left((G / H)^{K}\right) \otimes_{\mathbf{Q}(N(H) / H)} V_{H}
$$

on the objects of $\theta_{G}$. Given a morphism $\hat{a} \in E\left(G / K, G / K^{\prime}\right)$, define $\underline{V}_{H}(\hat{a})$ : $\underline{V}_{H}\left(G / K^{\prime}\right) \rightarrow \underline{V}_{H}(G / K)$ by

$$
\underline{V}_{H}(\hat{a})(\hat{b} \otimes v)=(\hat{b} \circ \hat{a}) \otimes v,
$$

where $\hat{b} \in(G / H)^{K^{\prime}}=E\left(G / K^{\prime}, G / H\right)$ and $v \in V_{H}$.

Proposition 3.2. $\underline{V}_{H}$ is projective in the category $\mathrm{Vec}_{G}$.

Proof. Let $M \stackrel{f}{\rightarrow} N \rightarrow 0$ be an exact sequence in $\operatorname{Vec}_{G}$ and $g: \underline{V}_{H} \rightarrow M$ a morphism. We have to construct a morphism $h: \underline{V}_{H} \rightarrow M$ such that the diagram

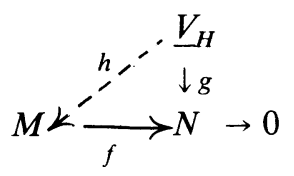


commutes. Since $V_{H}(G / H)=\mathbf{Q}(N(H) / H) \otimes_{\mathbf{Q}(N(H) / H)} V_{H}=V_{H}$, there exists an $N(H) / H$-map $h(G / H): V_{H} \rightarrow M(G / H)$ such that the diagram

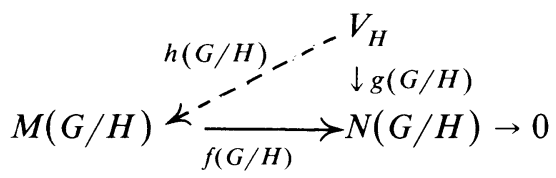

commutes. We define $h(G / K): \underline{V}_{H}(G / K) \rightarrow M(G / K)$ by

$$
h(G / K)(\hat{a} \otimes v)=M(\hat{a})(h(G / H)(v)),
$$

where $v \in V_{H}$ and $\hat{a} \in(G / H)^{K}=E(G / K, G / H)$.

COROLlary 3.3. $\mathbf{Q}(T)$ is projective in $\operatorname{Vec}_{G}$.

Proof. The $G$-set $T$ is the disjoint union of its orbits. Therefore, it suffices to prove that $\mathbf{Q}(G / H)$ is projective. But $\mathbf{Q}(G / H)$ is of the form $\underline{V}_{H}$, where $V_{H}=$ $\mathbf{Q}(N(H) / H)$.

Proposition 3.4. Every projective object $P$ in $\operatorname{Vec}_{G}$ can be written as $\oplus_{H \subseteq G} \underline{V}_{H}$.

Proof. In each conjugacy class $(H)$ of subgroups of $G$, we consider just one subgroup $H$. The subspace $\Sigma_{H^{\prime} \supset H} P\left(\hat{e}_{H, H^{\prime}}\right)\left(P\left(G / H^{\prime}\right)\right)$ of $P(G / H)$ is a $\mathbf{Q}(N(H) / H)$-submodule, where $\hat{e}_{H, H^{\prime}}: G / H \rightarrow G / H^{\prime}$ is the projection. Let $V_{H} \subseteq$ $P(G / H)$ be a $\mathbf{Q}(N(H) / H)$-submodule such that

$$
V_{H} \oplus \sum_{H^{\prime} \supset H} P\left(\hat{e}_{H, H^{\prime}}\right)\left(P\left(G / H^{\prime}\right)\right)=P(G / H) .
$$

We define $\underline{V}_{H}$ as above and $\underline{V}_{H_{i}}=0$ for any other subgroup conjugate to $H$.

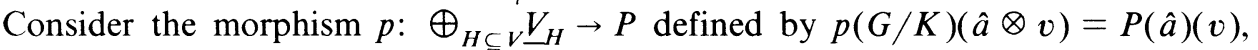
where $\hat{a} \in(G / H)^{K}$ and $v \in V_{H} \subseteq P(G / H)$. Clearly $p$ is an epimorphism. Since $P$ is projective, there exists $p^{\prime}: P \rightarrow \oplus_{H \subseteq G} \underline{V}_{H}$ such that $p \circ p^{\prime}=$ id. We shall show that $p$ is also a monomorphism by showing that $p^{\prime}$ is an epimorphism. It is clear that $p^{\prime}(G / G)$ is surjective. By using an inductive argument, it is easy to see that $p^{\prime}(G / H)$ is surjective after having assumed that $p^{\prime}\left(G / H^{\prime}\right)$ is surjective for every $H^{\prime} \supset H$.

Definition 3.5. A projective cover of a rational coefficient system $M$ is an epimorphism $p: P \rightarrow M$, where $P$ is projective in $\mathrm{Vec}_{G}$, with the following property: For any projective $P^{\prime}$ in $\operatorname{Vec}_{G}$ and any epimorphism $p^{\prime}: P^{\prime} \rightarrow M$ there exists an epimorphism $q: P^{\prime} \rightarrow P$ such that the diagram

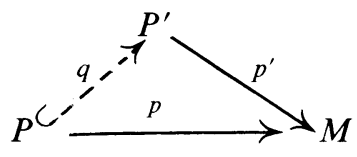

commutes.

Proposition 3.6. Any object $M$ in $\mathrm{Vec}_{G}$ has a projective cover which is unique up to isomorphism.

Proof. We construct $P=\oplus_{H \subseteq G} \underline{V}_{H}$ and $p: \bigoplus_{H \subseteq G \underline{V}_{H} \rightarrow M}$ as in the previous proposition. The space $V_{H}$ is the equivariant complement of 
$\Sigma_{H^{\prime} \supset H^{\prime}} M\left(\hat{e}_{H, H^{\prime}}\right)\left(M\left(G / H^{\prime}\right)\right) \subseteq M(G / H)$ or equal to 0 . Let $P^{\prime}$ be a projective in $\operatorname{Vec}_{G}$ and $p^{\prime}: P^{\prime} \rightarrow M$ an epimorphism. Since $P^{\prime}$ is projective there is a morphism $q$ : $P^{\prime} \rightarrow P$ such that the diagram

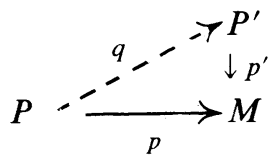

commutes. According to Proposition 3.4, we can write $P^{\prime}=\bigoplus_{H \subset G} \underline{W}_{H}$. Moreover, we can choose $W_{H}$ to be a subspace of $p^{\prime-1}(G / H)\left(V_{H}\right)$. Then $q(G / H)\left(W_{H}\right)=V_{H}$. Obviously $q(G / H)$ is surjective for every subgroup $H$. This completes the proof.

4. Systems of DGA's and the equivariant de Rham theorem. In this section we set up the right algebraic framework for the study of equivariant minimal models.

Recall that $\operatorname{Vec}_{G}^{*}$ is the category of covariant functors from $\theta_{G}$ to the category of rational vector spaces, or covariant rational coefficient systems for $G$. $\mathrm{Vec}_{G}^{*}$ is dual to $\mathrm{Vec}_{G}$. According to the results of the previous section, the injective objects in $\mathrm{Vec}_{G}^{*}$ are of the form $\bigoplus_{H \subseteq G} \underline{V}_{H}^{*}$; here $\underline{V}_{H}^{*}$ is defined by

$$
\underline{V}_{H}^{*}(G / K)=\operatorname{Hom}_{\mathbf{Q}(N(H) / H)}\left(\mathbf{Q}\left((G / H)^{K}\right), V_{H}^{*}\right),
$$

where $V_{H}^{*}$ is a right $\mathbf{Q}(N(H) / H)$-module. Moreover, any object $M$ in $\operatorname{Vec}_{G}^{*}$ has an injective envelope $I$, defined dually to the notion of a projective cover. In this case, $I=\bigoplus_{H \subseteq G} \underline{V}_{H}^{*}$, where $V_{H}^{*} \subseteq M(G / H)$ is defined by

$$
V_{H}^{*}=\bigcap_{H^{\prime} \supset H} \operatorname{ker} M\left(\hat{e}_{H, H^{\prime}}\right) \text {; }
$$

$\hat{e}_{H, H^{\prime}}: G / H \rightarrow G / H^{\prime}$ is the projection.

The most important example of an injective object in $\operatorname{Vec}_{G}^{*}$ is $\underline{\mathscr{E}}_{X}$, where $X$ is a $G$-complex, defined in the following way:

On a simplicial complex $X$, one can consider analogues of differential forms, the so-called $P L$ forms, which were first introduced by $\mathrm{H}$. Whitney [6]. Let

$$
\sigma^{n} \cong \Delta^{n}=\left\{\left(t_{0}, t_{1}, \ldots, t_{n}\right) \mid 0 \leqslant t_{i} \leqslant 1, \sum_{i=0}^{n} t_{i}=1\right\}
$$

be an $n$-simplex of $X$ canonically embedded in $R^{n+1}$. A $P L$ form of degree $p$ on $\sigma^{n}$ is a polynomial form

$$
\sum_{I} f_{I}\left(t_{0}, \ldots, t_{n}\right) d t_{i_{1}} \wedge \cdots \wedge d t_{i_{p}}
$$

where $I=\left\{i_{1}, \ldots, i_{p}\right\}$ and $f_{I}$ is a polynomial with coefficients in $\mathbf{Q}$. A global $P L$ form on $X$ is a collection of polynomial forms, one for each simplex of $X$, which coincide on common faces. The $P L$ forms of $X$ form a graded, commutative $\left(a \wedge b=(-1)^{|a||b|} b \wedge a\right)$, differential (the differential is of degree +1 ) algebra over $\mathbf{Q}$ (DGA), the so-called de Rham complex of $X$, denoted by $\varepsilon_{X}$.

4.2 Definition of $\underline{\varepsilon}_{X}$. Given a $G$-complex $X, \underline{\varepsilon}_{X}$ is a covariant functor from $\theta_{G}$ to the category of DGA's defined by $\underline{\varepsilon}_{X}(G / H)=\mathcal{E}_{X^{H}}$ on objects. If $\hat{a}: G / H \rightarrow G / H^{\prime}$ is a morphism in $\vartheta_{G}$ corresponding to $a \in G$, we define $\mathcal{E}_{X}(\hat{a})=a^{*}: \mathcal{E}_{X^{H}} \rightarrow \mathcal{E}_{X^{H}}$, where $a: X^{H^{\prime}} \rightarrow X^{H}$ is the map given by multiplication by $a$. 
We mentioned earlier that $\underline{C}_{*}(X)$ is a projective object in $\mathrm{Vec}_{G}$. In analogy with this we have the following result concerning $\mathscr{E}_{X}$ regarded as an object of $\mathrm{Vec}^{*}$ :

Proposition 4.3. $\underline{\varepsilon}_{X}$ is an injective object in $\mathrm{Vec}_{G}^{*}$.

Proof. We shall show $\underline{\mathcal{E}}_{X}=\bigoplus_{H \subseteq G} \underline{V}_{H}^{*}$, where $V_{H}^{*}=\bigcap_{H^{\prime} \supset H_{H}} \operatorname{ker} \underline{\mathcal{E}}_{X}\left(\hat{e}_{H, H^{\prime}}\right)$ for just one subgroup in each conjugacy class and $V_{H}^{*}=0$ otherwise. The set $\bigcap_{H^{\prime} \supset H} \operatorname{ker} \underline{\mathcal{E}}_{X}\left(\hat{e}_{H, H^{\prime}}\right) \subseteq \mathcal{E}_{X^{H}}$ is the set of $P L$ forms on $X^{H}$ which are equal to 0 when restricted to $X^{H^{\prime}}$ for every $H^{\prime} \supset H$.

We proceed inductively on the subgroups of $G$. We first construct a splitting $\sigma$ to $\mathcal{E}_{X} \rightarrow \mathcal{E}_{X^{G}}$ by considering a basis of the Q-vector space $\mathcal{E}_{X^{G}}$ and extending the $P L$ forms of this basis over $X$. We insist that the extended $P L$ forms vanish on each simplex of $X$ which is disjoint from $X^{G}$. This is possible by using the extension lemmas of [2]. Let $V_{G}^{*}=\sigma\left(\mathcal{E}_{X^{G}}\right) \cong \mathcal{E}_{X^{G}}$. The splitting $\sigma: \mathcal{E}_{X^{G}} \rightarrow \mathcal{E}_{X}$ induces splittings $\sigma_{H}: \mathcal{E}_{X^{G}} \rightarrow \mathcal{E}_{X^{H}}$ as well (compose $\sigma$ with the restriction map $\mathcal{E}_{X} \rightarrow \mathcal{E}_{X^{H}}$ ). Therefore

$$
\varepsilon_{X^{H}} \cong V_{G}^{*} \oplus \operatorname{ker}\left(\mathcal{E}_{X^{H}} \rightarrow \mathcal{E}_{X^{G}}\right)
$$

for every $H \subseteq G$. We can express this by $\underline{\mathcal{E}}_{X} \cong \underline{V}_{G}^{*} \oplus \underline{\mathcal{E}}_{X}^{\prime}$, where $\underline{\mathcal{E}}_{X}^{\prime}(G / H)=$ $\operatorname{ker}\left(\varepsilon_{X^{H}} \rightarrow \mathcal{E}_{X^{G}}\right)$.

Now consider $H \subseteq G$ and assume that

$$
\underline{\varepsilon}_{X}=\bigoplus_{H^{\prime}>H} \underline{V}_{H^{\prime}}^{*} \oplus \underline{\mathcal{E}}_{X, H},
$$

where $\underline{\mathcal{E}}_{X, H}(G / H)=\bigcap_{H \supset K, H^{\prime}>H} \operatorname{ker}\left(\mathcal{E}_{X^{K}} \rightarrow \mathcal{E}_{X^{H^{\prime}}}\right)$; the map in the parenthesis is induced by the inclusion. Here $H^{\prime}>H$ means that $H$ is conjugate to a subgroup of $H^{\prime}$ usually written $\left(H^{\prime}\right)>(H)$. Let $V_{H}^{*}=\bigcap_{H^{\prime} \supset H} \operatorname{ker} \underline{\varepsilon}_{X}\left(\hat{e}_{H, H^{\prime}}\right)$. Obviously $V_{H}^{*} \subseteq$ $\underline{\varepsilon}_{X, H}(G / H)$. By using the extension lemmas mentioned above, we construct a splitting $V_{H}^{*} \rightarrow \mathcal{E}_{X}$; we can extend the $P L$ forms of $V_{H}^{*}$ over $X$ in such a way that they vanish on each simplex of $X$ disjoint from $X^{H}$. By construction, two splittings $V_{H}^{*} \rightarrow \mathcal{E}_{X}$ and $V_{H^{\prime}}^{*} \rightarrow \mathcal{E}_{X}$ are disjoint if neither $(H) \leqslant\left(H^{\prime}\right)$ nor $\left(H^{\prime}\right) \leqslant(H)$. It is easy to check that

$$
\sum_{g \in G} V_{H}^{*} g^{*} \cong \operatorname{Hom}_{\mathbf{Q}(N(H) / H)}\left(\mathbf{Q}(G / H), V_{H}^{*}\right)
$$

and in general

$$
\left.\sum_{g \in G} V_{H}^{*} g^{*}\right|_{X^{K}} \cong \operatorname{Hom}_{\mathbf{Q}(N(H) / H)}\left(\mathbf{Q}\left((G / H)^{K}\right), V_{H}^{*}\right) ;
$$

$g^{*}$ means the operation on $P L$ forms induced by $g \in G$. This completes the inductive step of the proof.

Definition 4.4. Let $\mathrm{DGA}_{G}$ denote the category the objects of which are covariant functors from $\vartheta_{G}$ to the category of simple DGA's over $\mathbf{Q}$, which are injective when regarded as objects of $\mathrm{Vec}_{G}^{*}$; the morphisms are natural transformations between these functors.

We call such functors systems of DGA's for $G$.

For instance, $\underline{\mathscr{E}}_{X}$ is a system of DGA's for every $G$-complex $X$. In fact, the study of $\underline{\mathcal{E}}_{X}$ led us to the introduction of the category DGA ${ }_{G}$. 
We can consider cohomology of a system of DGA's $\mathfrak{A}$ in two ways.

(1) Let $\underline{H}^{*}(\mathfrak{A})$ be the covariant rational coefficient system defined by

$$
\underline{H}^{*}(\mathfrak{U})(G / H)=H^{*}(\mathfrak{U}(G / H)) .
$$

(2) The cohomology of $\mathfrak{A}$ with respect to a covariant rational coefficient system $M$ is defined by

$$
H^{*}(\mathfrak{A} ; M)=H^{*}(\operatorname{Hom}(M, \mathfrak{U}))
$$

$\operatorname{Hom}($,$) means morphisms in \mathrm{Vec}_{G}^{*}$ and is here a cochain complex (with grading and coboundary operator induced by the grading and differential of $\mathfrak{A})$.

As in the dual situation (2.2), there is a spectral sequence which relates the two cohomologies. We consider a projective resolution $M^{(*)}$ of $M$ in $\mathrm{Vec}_{G}^{*}$ and form the double complex $\operatorname{Hom}\left(M^{(*)}, \mathfrak{A}\right)$. Standard homological algebra applied to this double complex [4] yields a spectral sequence such that

$$
E_{2}^{s, t}=\operatorname{Ext}^{s}\left(M, \underline{H}^{t}(\mathfrak{A})\right) \Rightarrow H^{s+t}(\mathfrak{U} ; M) .
$$

The injectivity of $\mathfrak{A}$ is necessary for the convergence of the spectral sequence $\mathfrak{A}$. This spectral sequence is a central tool for our main constructions later on.

Notice that $H^{*}(\mathfrak{U} ; M)=\operatorname{Hom}\left(M, \underline{H}^{*}(\mathfrak{A})\right)$ if $M$ is projective. Moreover, a morphism $f: \mathfrak{A} \rightarrow \mathfrak{B}$ which induces an isomorphism $f^{*}: \underline{H}^{*}(\mathfrak{U}) \rightarrow \underline{H}^{*}(\mathfrak{B})$ also induces an isomorphism $f_{M}^{*}: H^{*}(\mathfrak{A} ; M) \rightarrow H^{*}(\mathfrak{B} ; M)$ for any $M \in \mathrm{Vec}_{G}^{*}$ and vice versa.

Imitating the nonequivariant case, we consider relative cohomology groups

$$
\underline{H}^{n}\left(\mathfrak{U}_{1}, \mathfrak{A}_{2}\right)=\underline{H}^{n}\left(\mathfrak{U}_{1}^{*} \times \mathfrak{U}_{2}^{*+1}\right)
$$

and

$$
H^{n}\left(\mathfrak{A}_{1}, \mathfrak{A}_{2} ; M\right)=H^{n}\left(\operatorname{Hom}\left(M, \mathfrak{A}_{1}^{*} \times \mathfrak{U}_{2}^{*+1}\right)\right),
$$

where $\mathfrak{A}_{2} \stackrel{i}{\hookrightarrow} \mathfrak{A}_{1}$ and $d: \mathfrak{A}_{1}^{n} \times \mathfrak{A}_{2}^{n+1} \rightarrow \mathfrak{A}_{1}^{n+1} \times \mathfrak{A}_{2}^{n+2}$ is equal to $\left(d_{1} \circ \mathrm{pr}_{1}+\mathrm{pr}_{2}\right.$, $\left.-d_{2} \circ \mathrm{pr}_{2}\right)$. Then there are long exact sequences

$$
\cdots \rightarrow \underline{H}^{n}\left(\mathfrak{U}_{2}\right) \stackrel{i^{*}}{\rightarrow} \underline{H}^{n}\left(\mathfrak{U}_{1}\right) \stackrel{j^{*}}{\rightarrow} \underline{H}^{n}\left(\mathfrak{U}_{1}, \mathfrak{A}_{2}\right) \stackrel{\delta^{*}}{\rightarrow} \underline{H}^{n+1}\left(\mathfrak{U}_{2}\right) \rightarrow \cdots
$$

and

$$
\begin{aligned}
\cdots & \rightarrow H^{n}\left(\mathfrak{A}_{2} ; M\right) \stackrel{i^{*}}{\rightarrow} H^{n}\left(\mathfrak{A}_{1} ; M\right) \stackrel{j^{*}}{\rightarrow} H^{n}\left(\mathfrak{A}_{1}, \mathfrak{A}_{2} ; M\right) \\
& \stackrel{\delta^{*}}{\rightarrow} H^{n+1}\left(\mathfrak{A}_{2} ; M\right) \rightarrow \cdots,
\end{aligned}
$$

where $j^{*}$ and $\delta^{*}$ are induced by $j=(\mathrm{id}, 0): \mathfrak{A}_{1}^{n} \rightarrow \mathfrak{U}_{1}^{n} \times \mathfrak{A}_{2}^{n+1}$ and $\mathrm{pr}_{2}: \mathfrak{A}_{1}^{n} \times \mathfrak{A}_{2}^{n+1} \rightarrow$ $\mathfrak{A}_{2}^{n+1}$ respectively.

If we take these facts into account, the proof of the equivariant de Rham theorem becomes straightforward.

Theorem 4.9. $H_{G}^{*}(X ; N) \cong H^{*}\left(\underline{\varepsilon}_{X} ; N^{*}\right)$, where $X$ is a $G$-complex, $N$ is any rational coefficient system in $\mathrm{Vec}_{G}$ and $N^{*}$ is its dual in $\mathrm{Vec}_{G}^{*}$.

Proof. We denote by $\underline{C}^{*}(X ; \mathbf{Q})$ the object in $\operatorname{Vec}_{G}^{*}$ defined by $\underline{C}^{*}(X ; \mathbf{Q})(G / H)=$ $C^{*}\left(X^{H} ; \mathbf{Q}\right)$, where the latter stands for the ordinary simplicial cochain complex of 
$X^{H}$ with coefficients in $\mathbf{Q}$. By integration of the $P L$ forms of $\mathcal{E}_{X^{H}}, H \subseteq G$, we get a morphism $i$ : $\underline{\varepsilon}_{X} \rightarrow \underline{C}^{*}(X ; \mathbf{Q})$, which is compatible with the differentials. Since $i(G / H): \mathcal{E}_{X^{H}} \rightarrow C^{*}\left(X^{H} ; \mathbf{Q}\right)$ is a cohomology isomorphism (simplicial de Rham theorem) for every $H \subseteq G, i$ induces an isomorphism on cohomology

$$
i^{*}: \underline{H}^{*}\left(\underline{\mathcal{E}}_{X}\right) \rightarrow \underline{H}^{*}\left(\underline{C}^{*}(X ; \mathbf{Q})\right)=\underline{H}^{*}(X ; \mathbf{Q}) .
$$

Consider the spectral sequences corresponding to the double complexes $\operatorname{Hom}\left(N^{*(*)}, \underline{\mathcal{E}}_{X}\right)$ and $\operatorname{Hom}\left(N^{*(*)}, \underline{C}^{*}(X ; \mathbf{Q})\right)$. Their $E_{2}$-terms are isomorphic:

$$
\operatorname{Ext}^{s}\left(N^{*}, \underline{H}^{t}\left(\mathcal{E}_{X}\right)\right) \cong \operatorname{Ext}^{s}\left(N^{*}, \underline{H}^{t}(X ; \mathbf{Q})\right) .
$$

On the other hand, $\operatorname{Ext}^{s}\left(N^{*}, \underline{H}^{t}(X ; \mathbf{Q})\right) \cong \operatorname{Ext}^{s}\left(\underline{H}_{t}(X ; \mathbf{Q}), N\right)$ because of duality. The first spectral sequence converges to $H^{*}\left(\underline{\mathscr{E}}_{X} ; N^{*}\right)$ and the second to $H_{G}^{*}(X ; N)$. This completes the proof.

We shall finish this section by defining the notions of homotopy between morphisms in $\mathrm{DGA}_{G}$ and homotopy equivalence between systems of DGA's.

As a motivation we remark the following: Let $f, g: X \rightarrow Y$ be two $G$-homotopic $G$-maps between $G$-complexes. In [1], it is proved that any $G$-map can be $G$-homotopically approximated by a simplicial $G$-map and any $G$-homotopy between two simplicial $G$-maps can be $G$-homotopically approximated by a simplicial $G$-homotopy. Therefore, we can assume without loss of generality that $f$ and $g$ are simplicial and that $H: X \times I \rightarrow Y$ is a simplical $G$-homotopy between them. The maps $f, g$ and $H$ induce homomorphisms on the de Rham complexes $\tilde{f}, \tilde{g}: \mathcal{E}_{Y} \rightarrow \mathcal{E}_{X}$ and $\tilde{H}$ : $\mathcal{E}_{Y} \rightarrow \mathcal{E}_{X \times I} \cong \mathcal{E}_{X} \otimes \mathbf{Q}(t, d t) ; \mathbf{Q}(t, d t)$ is the free DGA on two generators $t$ and $d t$ of degree 0 and 1 respectively with $d(t)=d t$. Moreover, $H_{0}=f$ and $H_{1}=g$ imply $e_{0} \circ \tilde{H}=\tilde{f}$ and $e_{1} \circ \tilde{H}=\tilde{g}$, where $e_{0}$ is the projection $\mathcal{E}_{X} \otimes \mathbf{Q}(t, d t) \rightarrow \mathcal{E}_{X}$ with $t=0, d t=0$ and $e_{1}$ the projection with $t=1, d t=0$. The restrictions of $f, g$ and $H$ on the fixed point sets $X^{K}$ and $X^{K} \times I$ induce homomorphisms $\mathcal{E}_{Y^{K}} \rightarrow \mathcal{E}_{X^{K}}$ and $\mathcal{E}_{Y^{K}} \rightarrow \mathcal{E}_{X^{K}} \otimes \mathbf{Q}(t, d t)$ as well, which have the above property. Furthermore, this procedure is functorial.

Given a system of DGA's $\mathfrak{A}$, let $\mathfrak{A}(t, d t)$ be the system of DGA's defined by $\mathfrak{A}(t, d t)(G / H)=\mathfrak{A}(G / H)(t, d t)$.

Definition 4.10. Two morphisms $f, g: \mathfrak{A} \rightarrow \mathfrak{B}$ are homotopic if there is a morphism $H: \mathfrak{A} \rightarrow \mathfrak{B}(t, d t)$ such that $e_{0} \circ H=f$ and $e_{1} \circ H=g$ where $e_{0}$ is the projection $t=0, d t=0$ and $e_{1}$ the projection $t=1, d t=0$.

Corollary 4.11. Any two simplicial G-homotopic G-maps $f, g: X \rightarrow Y$ induce homotopic morphisms $\tilde{f}, \tilde{g}: \mathcal{E}_{Y} \rightarrow \mathcal{E}_{X}$.

Proof. It follows directly from our discussion above.

As in the ordinary case, the homotopy relation defined in 4.10 is not an equivalence relation in the set of morphisms from $\mathfrak{A}$ to $\mathfrak{B}$. Instead, we have

Definition 4.12. Two systems of DGA's $\mathfrak{A}$ and $\mathfrak{B}$ are weakly equivalent if there is a chain of cohomology isomorphisms

$$
\mathfrak{U} \stackrel{f_{1}}{\rightarrow} \mathfrak{E}_{1} \stackrel{f_{2}}{\rightarrow} \mathfrak{c}_{2} \leftarrow \cdots \rightarrow \mathfrak{B}
$$

connecting them. 
By means of the minimal systems of DGA's, which will be introduced in the next section, we shall prove that weak equivalence classes of systems of DGA's for $G$ correspond bijectively to rational homotopy types of $G$-spaces.

5. Minimal systems of DGA's. Recall that a (connected) DGA $\Re$ is minimal if it is free and its differential is decomposable. Freeness means that $\mathfrak{N}=\mathfrak{N}_{1}$ $\otimes \cdots \otimes \pi_{n} \otimes \cdots$, where

$$
\Re_{n}=\left\{\begin{array}{l}
\text { a polynomial algebra generated by elements of degree } p, \quad p \text { even } \\
\text { an exterior algebra generated by elements of degree } p, \quad p \text { odd }
\end{array}\right.
$$

and decomposability of the differential $d$ means that $d(\mathscr{T}) \subseteq \Re^{+} \wedge \mathscr{T}^{+}$, where

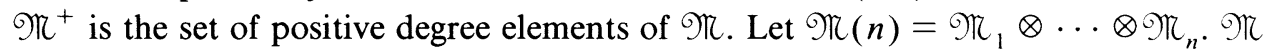
is called simple if $d$ restricted to $\Re(n)$ takes values in $\Re(n-1)$ for every $n$. We restrict attention to simple DGA's, i.e., to DGA's the minimal model of which is simple.

Definition 5.1. A system of DGA's $\mathfrak{M}$ is minimal if it satisfies the following properties:

(i) $\mathfrak{M}(G / H)$ is a free DGA for all $H \subseteq G$,

(ii) $\mathfrak{M}(G / G)$ is a minimal DGA (initial condition), and

(iii) $d\left(\cap_{H^{\prime} \supset H} \operatorname{ker} \mathfrak{M}\left(\hat{e}_{H, H^{\prime}}\right)\right) \subseteq \mathfrak{M}(G / H)$ is decomposable, where $\hat{e}_{H, H^{\prime}}: G / H \rightarrow$ $G / H^{\prime}$ is the projection.

We shall show that the minimal systems of DGA's for $G$ play the same role in the category of $\mathrm{DGA}_{G}$ as the minimal DGA's in the category of DGA's. In this section, we shall state the results and in $\S 7$, we shall prove them.

THEOREM 5.2. If $f: \mathfrak{M} \rightarrow \mathfrak{R}$ is a cohomology isomorphism between systems of DGA's, then $f$ is an isomorphism.

THEOREM 5.3. For any system of DGA's $\mathfrak{A}$ there exist a minimal system of DGA's $\mathfrak{M}$ and a morphism $\rho: \mathfrak{M} \rightarrow \mathfrak{A}$ inducing an isomorphism on cohomology.

The following propositions describe the properties of minimal systems of DGA's with respect to homotopy. They are completely analogous to the nonequivariant ones.

Proposition 5.4. The homotopy relation in the set of morphisms from a minimal system of DGA's to an arbitrary system of DGA's is an equivalence relation.

Proposition 5.5. Let $\pi: \mathfrak{A} \rightarrow \mathfrak{B}$ be a cohomology isomorphism and let $f: \mathfrak{M} \rightarrow \mathfrak{B}$ be an arbitrary morphism, where $\mathfrak{M}$ is minimal. Then there is a morphism $f^{\prime}: \mathfrak{M} \rightarrow \mathfrak{A}$, unique up to homotopy, such that the diagram

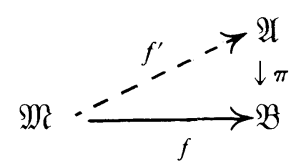

commutes up to homotopy. 
COROllaRY 5.6. If $\pi: \mathfrak{A} \rightarrow \mathfrak{B}$ is a cohomology isomorphism, then it induces a bijection

$$
\pi_{*}:[\mathfrak{M}, \mathfrak{A}] \rightarrow[\mathfrak{M}, \mathfrak{B}],
$$

where $[\mathfrak{M}, \mathfrak{A}]$ is the set of homotopy classes of morphisms from $\mathfrak{M}$ to $\mathfrak{A}$.

CoRollaRy 5.7. Let $\rho: \mathfrak{M} \rightarrow \mathfrak{A}$ and $\rho^{\prime}: \mathfrak{M}^{\prime} \rightarrow \mathfrak{A}$ be two cohomology isomorphisms, where $\mathfrak{M}$ and $\mathfrak{M}^{\prime}$ are minimal systems of $D G A$ 's. Then there is an isormorphism $i$ : $\mathfrak{M} \rightarrow \mathfrak{M}^{\prime}$ such that the diagram

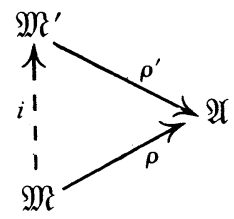

commutes up to homotopy.

This uniqueness result makes the following definition meaningful.

DEFINITION 5.8. Let $\rho: \mathfrak{M} \rightarrow \mathfrak{U}$ be a cohomology isomorphism, where $\mathfrak{M}$ is minimal. Then $\mathfrak{M}$ is called the minimal model of $\mathfrak{A}$.

COROLLARY 5.9. In each weak equivalence class of systems of DGA's for $G$ there is one and, up to isomorphism, only one minimal system of DGA's.

The following construction is central to the analysis of minimal systems of DGA's and mirrors the topological construction of principal $G$-fibrations (see Lemmas 6.3 and 6.4 below). It is an important tool in the proof of Theorem 5.3.

Construction 5.10. Let $\mathfrak{A}$ be a system of DGA's for $G$, let $W_{n}$ be an object in $\operatorname{Vec}_{G}^{*}$ and let $d: W_{n} \rightarrow \underline{Z}^{n+1}(\mathfrak{A})$ be a morphism in Vec $\operatorname{Ver}_{G}^{*}$, where $\underline{Z}^{n+1}(\mathfrak{A})(G / H)$ is the set of cocycles of degree $n+1$ of the DGA $\mathfrak{A}(G / H)$. We construct a new system of DGA's $\mathfrak{A}\left(W_{n}\right)$, called the elementary extension of $\mathfrak{A}$ with respect to $d$, as follows. Let

$$
0 \rightarrow W_{n} \stackrel{w}{\rightarrow} W_{n, 0} \stackrel{w_{0}}{\rightarrow} W_{n, 1} \rightarrow \cdots W_{n, i} \stackrel{w_{i}}{\rightarrow} \cdots
$$

be a minimal injective resolution of $W_{n}$ in $\mathrm{Vec}_{G}^{*}$, i.e., an injective resolution such that $W_{n, i}$ is the injective envelope of im $w_{i-1}$. One can see immediately that $d$ induces morphisms $d_{i}: W_{n, i} \rightarrow \mathfrak{A}^{n+i+1}$ such that the diagram

$$
\begin{aligned}
& 0 \rightarrow \quad W_{n} \quad \stackrel{w}{\rightarrow} \quad W_{n, 0} \stackrel{\stackrel{w_{0}}{\rightarrow}}{\rightarrow} W_{n, 1} \quad \rightarrow \quad \ldots \quad W_{n, i} \stackrel{w_{i}}{\rightarrow} \quad \ldots
\end{aligned}
$$

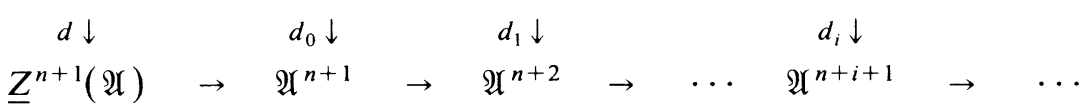

commutes. Let $W_{n, *}=\bigoplus_{m \geqslant 0} W_{n, i}$, where $W_{n, i}$ is considered as being concentrated in degree $n+i$. Define an object $\Lambda\left(W_{n, *}\right)$ in $\operatorname{Vec}_{G}^{*}$ by

$$
\Lambda\left(W_{n, *}\right)(G / H)=\Lambda\left(W_{n, *}(G / H)\right),
$$

where the latter is the free graded commutative algebra generated by the graded vector space $W_{n, *}(G / H)$. We define $\mathfrak{A}\left(W_{n}\right)=\mathfrak{A} \otimes \Lambda\left(W_{n, *}\right)$, where the differential 
of $\mathfrak{U}\left(W_{n}\right)$ restricts on $\mathfrak{U}$ to the given differential and restricts on $W_{n, i}$ to $w_{i}+(-1)^{i} d_{i}$, $i \geqslant 0$. The object $\mathfrak{A}\left(W_{n}\right)$ is injective as it is the tensor product of injectives.

REMARK. The injective resolution of $W_{n}$ corresponds by duality to the projective resolution in the construction of Eilenberg-Mac Lane $G$-complexes.

As an immediate corollary of the proof of Theorem 5.3 we shall have the following description of minimal systems of DGA's:

COROLlaRY 5.11. Any minimal system of DGA's $\mathfrak{M}$ can be written as a union of an expanding sequence

$$
\mathfrak{M}(1) \subseteq \cdots \subseteq \mathfrak{M}(n-1) \subseteq \mathfrak{M}(n) \subseteq \cdots
$$

of minimal systems of DGA's, where $\mathfrak{M}(n)$ is an elementary extension $\mathfrak{M}(n)=$ $\mathfrak{M}(n-1)\left(W_{n}\right)$. Moreover,

$$
W_{n} \cong \underline{H}^{n}(\mathfrak{M}(n), \mathfrak{M}(n-1))
$$

6. Equivariant minimal models: the main theorems. In this section we establish the relation between $G$-spaces and minimal systems of DGA's for $G$.

DeFinition 6.1. The equivariant minimal model $\mathfrak{M}_{X}$ of a $G$-complex $X$ is the minimal model of the system of DGA's $\underline{\varepsilon}_{X}$.

The main results of this paper are the following Theorems 6.2 and 6.5:

THEOREM 6.2. The correspondence $X \mapsto \mathfrak{M}_{X}$ induces a bijection between equivariant rational homotopy types of $G$-spaces on the one hand and isomorphism classes of minimal systems of DGA's for $G$ on the other. Moreover, the following relations hold.

(1) $H_{G}^{*}(X ; N) \cong H^{*}\left(\underline{\mathcal{E}}_{X} ; N^{*}\right)$, where $N$ is a coefficient system in $\operatorname{Vec}_{G}$ and $N^{*}$ is its dual in $\mathrm{Vec}_{G}^{*}$.

(2) $\underline{\pi}_{n}(X) \otimes \mathbf{Q} \cong W_{n}^{*}$, where $\mathfrak{M}_{X}(n)=\mathfrak{M}_{X}(n-1)\left(W_{n}\right)$ and $W_{n}^{*}$ is the dual of $W_{n}$ in $\mathrm{Vec}_{G}$.

(3) If

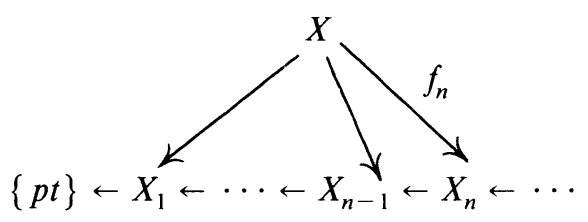

is an equivariant Postnikov decomposition of $X$ then

(i) $\mathfrak{M}_{X}(n)$ is the equivariant minimal model of $X_{n}$ and

(ii) the morphism

$$
W_{n} \stackrel{d}{\rightarrow} \underline{Z}^{n+1}\left(\mathfrak{M}_{X}(n-1)\right)
$$

determines the equivariant $k$-invariant

$$
k^{n+1} \in H_{G}^{n+1}\left(X_{n-1} ; \underline{\pi}_{n}(X)\right) \otimes \mathbf{Q} \cong H_{G}^{n+1}\left(X_{0, n-1} ; \underline{\pi}_{n}\left(X_{0}\right)\right)
$$

of the equivariant rationalization $X_{0}$ of $X$.

Proof. Relation (1) is an immediate consequence of the equivariant de Rham theorem. 
The proof of the bijection stated in the theorem is based on the following two lemmas. They establish the equivalence between elementary extensions of systems of DGA's and principal $G$-fibrations. We shall state them here and prove them in $\S 8$.

Say that a system of DGA's $\mathfrak{A}$ is geometric for $X$ if there is a cohomology isomorphism $\mathfrak{A} \rightarrow \underline{\mathcal{E}}_{X}$.

Lemma 6.3 (analogue to the Hirsch lemma). Let $F=K(M, n) \rightarrow E \stackrel{p}{\rightarrow} X$ be $a$ $G$-fibration and let $\mathfrak{A}$ be a system of DGA's geometric for $X$. Then there is an elementary extension $\tilde{\mathfrak{U}}=\mathfrak{A}\left(W_{n}\right)$ of $\mathfrak{U}$ which is geometric for E. Moreover, $W_{n}=$ $\underline{H}^{n}(F ; \mathbf{Q})$.

Lemma 6.4 (converse to the previous one). Let $X$ be a G-complex and let $\mathfrak{A}$ be a system of DGA's geometric for $X$. If $\mathfrak{\mathfrak { A }}$ is an elementary extension of $\mathfrak{U}$ with respect to $\tilde{d}: W_{n} \rightarrow \underline{Z}^{n+1}(\mathfrak{U})$ there is a $G$-fibration $K\left(W_{n}^{*}, n\right) \rightarrow E \rightarrow X$ such that $\tilde{\mathfrak{A}}$ is geometric for $E$.

Let $\mathfrak{M}$ be a minimal system of DGA's for $G$. Then there is an expanding sequence of elementary extensions

$$
\underline{\mathbf{Q}} \subseteq \mathfrak{M}(1) \subseteq \cdots \subseteq \mathfrak{M}(n-1) \subseteq \mathfrak{M}(n) \subseteq \cdots
$$

such that $\mathfrak{M}=\cup_{n \geq 0} \mathfrak{M}(n)$ (Corollary 5.11). According to the previous lemma, we can construct inductively (starting from a point) a sequence of principal $G$-fibrations, i.e., an equivariant Postnikov decomposition

$$
\{p t\} \leftarrow X_{1} \leftarrow \cdots \leftarrow X_{n-1} \leftarrow X_{n} \leftarrow \cdots .
$$

The inverse limit of it is a $G$-space. The barycentric subdivision of the semisimplicial resolution of this space is a $G$-complex.

Conversely, let $X$ be a $G$-complex. According to the first lemma, the $G$-Postnikov decomposition of $X$ induces an expanding sequence of elementary extensions of minimal systems of DGA's for $G$ (starting from $\mathbf{Q}, \mathbf{Q}(G / H)=\mathbf{Q}$ ). Moreover, the properties (2) and (3) of the theorem are valid by construction. The uniqueness arguments are analogous to the nonequivariant ones and are based on 2.5 and 5.7.

TheOREM 6.5. There exists a bijection $[X, Y]_{G} \cong\left[\mathfrak{M}_{Y}, \mathfrak{M}_{X}\right]$, where $X$ is a G-space and $Y$ is a $\mathbf{Q}-G$-space.

The proof consists of the following two propositions.

Proposition 6.6. Let $f: X \rightarrow Y$ be a G-map between two G-complexes and let $\rho_{X}$ : $\mathfrak{M}_{X} \rightarrow \underline{\mathcal{E}}_{X}$ and $\rho_{Y}: \mathfrak{M}_{Y} \rightarrow \underline{\mathcal{E}}_{Y}$ be equivariant minimal models of $X$ and $Y$ respectively. Then there exists a morphism $\tilde{f}: \mathfrak{M}_{Y} \rightarrow \mathfrak{M}_{X}$ such that the diagram

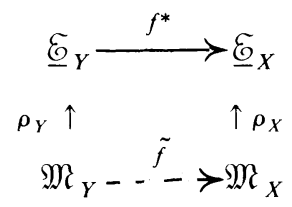

commutes up to homotopy. Moreover, $\tilde{f}$ is unique up to homotopy and depends only on the rational G-homotopy class of $f$. 
Proof. This is an immediate consequence of Corollary 4.11 and Proposition 5.5. Just as in the nonequivariant case, the last statement follows from a relative version of Proposition 5.5.

Proposition 6.7. Let $\tilde{f}: \mathfrak{N} \rightarrow \mathfrak{M}$ be a morphism between two minimal systems of $D G A$ 's for $G$, where $\mathfrak{M}$ and $\mathfrak{N}$ are the equivariant minimal models of the rational $G$-spaces $X$ and $Y$ respectively. Then there is a $G$-map $f: X \rightarrow Y$, such that the diagram

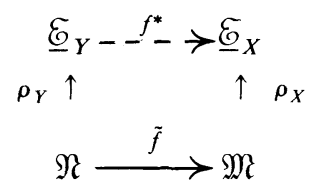

commutes up to homotopy. Moreover, $f$ is unique up to homotopy and depends only on the homotopy class of $\tilde{f}$.

The proof is deferred to $\S 8$.

\section{Deferred proofs from $\$ 5$.}

Proof of Proposition 5.2. Let $f: \mathfrak{M} \rightarrow \mathfrak{N}$ be a cohomology isomorphism. Since $\mathfrak{M}(G / G)$ and $\mathfrak{R}(G / G)$ are minimal DGA's and $f(G / G)$ is a cohomology isomorphism between them, $f(G / G)$ is an isomorphism. Given $H \subseteq G$, assume inductively that $f\left(G / H^{\prime}\right): \mathfrak{M}\left(G / H^{\prime}\right) \rightarrow \mathfrak{R}\left(G / H^{\prime}\right)$ is an isomorphism for all subgroups $H^{\prime} \supset H$. We shall prove that $f(G / H)$ is also an isomorphism. Let $f(G / H)$ be an isomorphism when restricted to $\cup_{k \leqslant n-1} \mathfrak{M}(G / H)^{k}$.

Injectivity of $f(G / H)^{n}: \mathfrak{M}(G / H)^{n} \rightarrow \mathfrak{R}(G / H)^{n}$. Let $x \in \mathfrak{M}(G / H)^{n}$ be such that $f(G / H)(x)=0$. From the injectivity of $f\left(G / H^{\prime}\right), H^{\prime} \supset H$, and the relation $f\left(G / H^{\prime}\right) \circ \mathfrak{M}\left(\hat{e}_{H, H^{\prime}}\right)(x)=\mathfrak{R}\left(\hat{e}_{H, H^{\prime}}\right) \circ f(G / H)(x)=0$ it follows that $\mathfrak{M}\left(\hat{e}_{H, H^{\prime}}\right)(x)$ $=0$ for every $H^{\prime} \supset H$. Therefore $d x \in \mathfrak{M}(G / H)^{+} \cdot \mathfrak{M}(G / H)^{+}$, i.e., $d x$ can be expressed as a sum of products of elements of degree less than $n$. Since $f(G / H)$ is injective in lower degrees and the algebras are free, we have $d x=0$. Because of the injectivity of $f(G / H)^{*}$ on the cohomology we have $x=d t, t \in \mathfrak{M}(G / H)^{n-1}$. Then $d f(G / H)(t)=f(G / H)(d t)=0$, so $f(G / H)(t)$ is the cocycle. The surjectivity of $f(G / H)^{*}$ on cohomology implies that there is a cocycle $t^{\prime} \in \mathfrak{M}(G / H)^{n-1}$ such that $f(G / H)(t)-f(G / H)\left(t^{\prime}\right)=d u, u \in \mathfrak{R}(G / H)^{n-2}$. Therefore we can assume that

$$
f(G / H)(t)=d u, \quad u \in \mathfrak{R}(G / H)^{n-2} .
$$

Since $f(G / H)$ is an isomorphism in degree $n-2$, there is $u^{\prime} \in \mathfrak{M}(G / H)^{n-2}$ such that $f(G / H)\left(u^{\prime}\right)=u$. Then $d u^{\prime}$ is a preimage of $f(G / H)(t)$ and, because of the injectivity of $f(G / H)$ in degree $n-1$, it must be equal to $t$. Then $d t=d d u^{\prime}=0$, i.e., $x=0$.

Surjectivity of $f(G / H)^{n}$. Let $y \in \Re(G / H)^{n}$. We shall show that $y=f(G / H)(x)$, $x \in \mathfrak{M}(G / H)^{n}$. Let $y_{H^{\prime}}=\mathfrak{N}\left(\hat{e}_{H, H^{\prime}}\right)(y) \in \mathfrak{R}\left(G / H^{\prime}\right), H^{\prime} \supset H$, and consider the preimages $\bar{y}_{H^{\prime}} \in \mathfrak{M}(G / H)^{n}$.

Claim. There is an element $\bar{y} \in \mathfrak{M}(G / H)^{n}$ such that

$$
\mathfrak{M}\left(\hat{e}_{H, H^{\prime}}\right)(\bar{y})=\bar{y}_{H^{\prime}} \quad \text { for all } H^{\prime} \supset H .
$$


Granting the claim, replace $y$ by $y-f(G / H)(\bar{y})$ and so assume that $\mathfrak{R}\left(\hat{e}_{H, H^{\prime}}\right)(y)=0$ for every $H^{\prime} \supset H$. Then $d y$ is decomposable and it has a preimage $z, f(G / H)(z)=d y$, because $f(G / H)$ is surjective in lower degrees. Moreover, $z$ and $d z$ are decomposable, $n$ being the highest degree of indecomposable elements appearing in the expression of $d z$. Since $f(G / H)(d z)=0$ and $f(G / H)$ is injective in degrees less than or equal to $n, d z=0$. Moreover, $z$ is a coboundary because $f^{*}(G / H)$ is injective. Let $z=d v, v \in \mathfrak{M}(G / H)^{n}$. If we replace $y$ by $y-f(G / H)(v)$ we can assume $d y=0$. Then there is a $y^{\prime} \in \mathfrak{M}(G / H)^{n}$ such that $f(G / H)\left(y^{\prime}\right)-y=d u$. So we can assume

$$
y=d u, \quad u \in \mathfrak{N}(G / H)^{n-1} .
$$

But $u$ has a preimage $u^{\prime} \in \mathfrak{M}(G / H)^{n-1}$. Then $d u^{\prime}$ is a preimage of $y$, i.e., $f(G / H)\left(d u^{\prime}\right)=y$.

PROOF OF THE ClAIM. $\mathfrak{M}^{n}$ is injective by assumption. Therefore we can write $\mathfrak{M}^{n}=\bigoplus_{K \subseteq G} V_{K}^{*}$. In particular,

$$
\mathfrak{M}^{n}(G / H)=\bigoplus_{K \subseteq G} \underline{V}_{K}^{*}(G / H)=V_{H}^{*} \underset{H^{\prime}>H}{\bigoplus} \operatorname{Hom}_{\mathbf{Q}\left(N\left(H^{\prime}\right) / H^{\prime}\right)}\left(\mathbf{Q}\left(\left(G / H^{\prime}\right)^{H}\right), V_{H^{\prime}}^{*}\right),
$$

because $(G / K)^{H}=0$ if $H$ is not conjugate to a subgroup of $K$. Since $f\left(G / H^{\prime}\right)$ is an isomorphism for all $H^{\prime}>H$ and $f(G / H)^{n}: \mathfrak{M}(G / H)^{n} \rightarrow \mathfrak{R}(G / H)^{n}$ is injective we can write

$$
\mathfrak{R}^{n}(G / H)=V_{H}^{*} \underset{H^{\prime}>H}{\bigoplus} \operatorname{Hom}_{\mathbf{Q}\left(N\left(H^{\prime}\right) / H^{\prime}\right)}\left(\mathbf{Q}\left(\left(G / H^{\prime}\right)^{H}\right), V_{H^{\prime}}^{*}\right) \oplus W_{H}^{*}
$$

Moreover, we can choose $W_{H}^{*}$ such that $W_{H}^{*} \subseteq \bigcap_{H^{\prime} \supset H} \operatorname{ker} \mathfrak{R}\left(\hat{e}_{H, H^{\prime}}\right)$ using the fact that $\Re^{n}$ is injective. Consider the projection of $y$ on

$$
V_{H}^{*} \underset{H^{\prime}>H}{\bigoplus} \operatorname{Hom}_{\mathbf{Q}\left(N\left(H^{\prime}\right) / H^{\prime}\right)}\left(\mathbf{Q}\left(\left(G / H^{\prime}\right)^{H}\right), V_{H^{\prime}}^{*}\right) .
$$

It has a preimage $\bar{y}$ in $\mathfrak{M}^{n}(G / H)$. Then $\mathfrak{M}\left(\hat{e}_{H, H^{\prime}}\right)(\bar{y})=\bar{y}_{H^{\prime}}$ for all $H^{\prime} \supset H$.

Proof of Theorem 5.3. (Existence of the minimal model): We proceed inductively on degree. Suppose there is a minimal system of DGA's $\mathfrak{M}(n-1)$ and a morphism $\rho_{n-1}: \mathfrak{M}(n-1) \rightarrow \mathfrak{A}$ such that $\rho_{n-1}^{k}: \underline{H}^{k}(\mathfrak{M}(n-1)) \rightarrow \underline{H}^{k}(\mathfrak{A})$ is an isomorphism if $k \leqslant n-1$ and a monomorphism if $k=n$.

We consider $W_{n}^{\prime}=\underline{\text { coker } \rho_{n-1}^{n} \text { in Vec }}$, where

$$
\text { coker } \rho_{n-1}^{n}(G / H)=\text { coker } \rho_{n-1}^{n}(G / H) \text {. }
$$

Let $p: P \rightarrow W_{n}^{\prime}$ be an epimorphism, where $P$ is projective. There exist liftings $p^{\prime}$ and $p^{\prime \prime}$ in the following diagram by projectivity; here $\underline{Z}^{n}(\mathfrak{H})$ is the functor in $\operatorname{Vec}_{G}^{*}$ defined by $\underline{Z}^{*}(\mathfrak{A})(G / H)=Z^{n}(\mathfrak{A}(G / H))$, where $Z^{n}()$ means the set of cocycles of degree $n$.

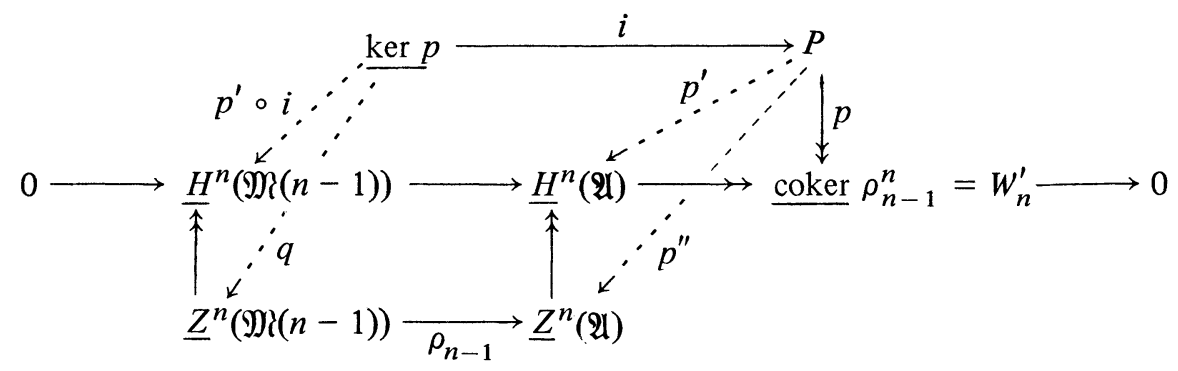


The existence of the morphism $q$, such that the entire diagram commutes, follows from the existence of $p^{\prime}$ and $p^{\prime \prime}$ by comparing the two spectral sequences

$$
E_{2}^{s, t}=\operatorname{Ext}^{s}\left(\underline{H}^{t}(\mathfrak{M}(n-1)), \underline{\operatorname{ker}} p\right) \stackrel{\rho_{n-1}^{*}}{\rightarrow} \operatorname{Ext}^{s}\left(\underline{H}^{t}(\mathfrak{A}), \underline{\operatorname{ker}} p\right)=E_{2}^{s, t} .
$$

Because of our assumption on $\rho_{n-1}$, we have $E_{2}^{s, t} \cong E_{2}^{\prime s, t}$ for every $s$ and for $t \leqslant n-1$. Moreover, $E_{2}^{0, n} \subseteq{E^{\prime}}_{2}^{0, n}$ and $\left.{d^{\prime}}_{r}^{0, n}\right|_{E_{r}^{0, n}}=d_{r}^{0, n}$ for every $r$. Thus

$$
E_{\infty}^{s, t} \cong E_{\infty}^{\prime s, t}
$$

for $s+t \leqslant n, s \neq 0$ and $E_{\infty}^{0, n} \subseteq E_{\infty}^{\prime 0, n}$. In particular, we consider the edge homomorphisms

$$
\begin{array}{ccccccccc}
H^{n} & \rightarrow & H^{n} / F^{\prime} H^{n} & = & E_{\infty}^{0, n} \subseteq & \cdots & \subseteq E_{3}^{0, n} & \subseteq & E_{2}^{0, n} \\
\downarrow & & & & \cap & & \cap & & \cap \\
H^{\prime n} & \rightarrow & H^{\prime n} / F^{\prime} H^{\prime n} & = & E_{\infty}^{\prime, n} \subseteq & \cdots & \subseteq E_{3}^{\prime 0, n} & \subseteq & E_{2}^{\prime 0, n}
\end{array}
$$

where $H^{n}$ and $H^{\prime n}$ are equal to $H^{n}(\mathfrak{M}(n-1)$, ker $p)$ and $H^{n}(\mathfrak{A}, \operatorname{ker} p)$ respectively. The isomorphism (7.1) implies $F^{i} H^{n}=F^{i} H^{\prime n}$ for every $i \geqslant 1$ by induction; therefore, $H^{n} \subseteq H^{n}$.

Consider $\left[p^{\prime} \circ i\right] \in E_{2}^{0, n} \subseteq E_{2}^{\prime 0, n}$. Since $\left[p^{\prime} \circ i\right]$ has a preimage $\left[p^{\prime \prime} \circ i\right] \in H^{\prime n}$, it belongs to $E_{\infty}^{\prime 0, n}$. This means that $d_{r}^{\prime}\left(\left[p^{\prime} \circ i\right]\right)=0$ for every $r$. But then also $d_{r}\left(\left[p^{\prime} \circ i\right]\right)=0$ for every $r$. Therefore $\left[p^{\prime} \circ i\right]$ has a preimage $[q] \in H^{n}$. The difference between $p^{\prime \prime} \circ i$ and $\rho_{n-1} \circ q$ by a coboundary can be easily corrected.

Since $\mathfrak{M}(n-1)^{n}$ is injective in $\operatorname{Vec}_{G}^{*}$, the morphism $q:$ ker $p \rightarrow \underline{Z}^{n}(\mathfrak{M}(n-1)) \subseteq$ $\mathfrak{M}(n-1)^{n}$ can be extended to a morphism $\left.q^{\prime}: P \rightarrow \mathfrak{M} \overline{(n}-1\right)^{n}$. The morphisms $d \circ q^{\prime}: P \rightarrow \mathfrak{M}(n-1)^{n+1}$ and $\rho_{n-1} \circ q^{\prime}-p^{\prime \prime}: P \rightarrow \mathfrak{A}^{n}$ are equal to 0 when restricted to ker $p$. Therefore they factor through $W_{n}^{\prime}$. We call the new morphisms $d^{\prime}$ : $W_{n}^{\prime} \rightarrow \mathfrak{M}(n-1)^{n+1}$ and $\rho_{n}^{\prime}: W_{n}^{\prime} \rightarrow \mathfrak{U}^{n}$ respectively.

As in construction 5.10, we consider a minimal injective resolution of $W_{n}^{\prime}$ in $\operatorname{Vec}_{G}^{*}$

$$
0 \rightarrow W_{n}^{\prime} \stackrel{w^{\prime}}{\rightarrow} W_{n, 0}^{\prime} \stackrel{w_{0}^{\prime}}{\rightarrow} W_{n, 1}^{\prime} \stackrel{w_{1}^{\prime}}{\rightarrow} \cdots W_{n, i}^{\prime} \stackrel{w_{i}^{\prime}}{\rightarrow} \cdots
$$

and construct an elementary extension $\mathfrak{M}(n-1)\left(W_{n}^{\prime}\right)$ of $\mathfrak{M}(n-1)$ with respect to $d^{\prime}: W_{n}^{\prime} \rightarrow \mathfrak{M}(n-1)^{n+1}$. Then $\rho_{n}^{\prime}$ induces morphisms $\rho_{n, i}^{\prime}: W_{n, i}^{\prime} \rightarrow \mathfrak{A}^{n+1}$ compatible with the differential of $\mathfrak{M}(n-1)\left(W_{n}^{\prime}\right)$. Therefore we can extend $\rho_{n-1}$ and $\rho_{n}^{\prime}$ to a morphism between systems of DGA's which we call again $\rho_{n}^{\prime}: \mathfrak{M}(n-1)\left(W_{n}^{\prime}\right) \rightarrow \mathfrak{A}$. It is easy to check that this morphism induces an isomorphism on $\underline{H}^{n}$. In order to do so, we consider the effect of the above construction on each $G / H, H \subseteq G$, and so reduce the verification to the nonequivariant case.

Now let $W_{n}^{\prime \prime}$ be the kernel of $\rho_{n}^{\prime n+1}: H^{n+1}\left(\mathfrak{M}(n-1)\left(W_{n}^{\prime}\right)\right) \rightarrow H^{n+1}(\mathfrak{U})$ and let $q$ : $Q \rightarrow W_{n}^{\prime \prime}$ be an epimorphism, where $Q$ is a projective object in $\operatorname{Vec}_{G}^{*}$. In the diagram

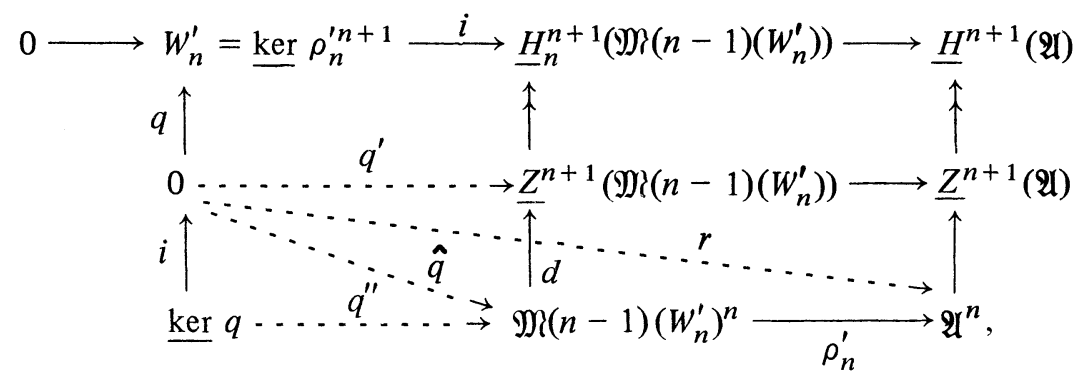


$q^{\prime}$ exists because $\mathbf{Q}$ is projective. We claim that the morphism $q^{\prime} \circ i$ : ker $q \rightarrow \underline{Z}^{n+1}\left(\mathfrak{M}(n-1)\left(W_{n}^{\prime}\right)\right)$ is a coboundary with respect to the cohomology $\overline{H^{n}+1}\left(\mathfrak{M}(n-1)\left(w_{n}^{\prime}\right) ; \operatorname{ker} q\right)$, i.e., it factors through the differential $d$ via some $q^{\prime \prime}$ : $\operatorname{ker} q \rightarrow \mathfrak{M}(n-1)\left(W_{n}^{\prime}\right)^{n}$. Indeed, the morphism $\rho_{n}^{\prime} \circ q^{\prime}$ can be regarded as $\rho_{n}^{\prime} \circ q^{\prime}$ : $\bar{Q} \rightarrow \underline{B}^{n+1}(\mathfrak{A})$, where $\underline{B}^{n+1}(\mathfrak{A})(G / H)$ is the set of coboundaries of degree $n+1$ of the DGA $\mathfrak{A}(G / H)$. Since $d: \mathfrak{U}^{n} \rightarrow \underline{B}^{n+1}(\mathfrak{A})$ is an endomorphism, there exists $r$ : $Q \rightarrow \mathfrak{A}^{n}$ such that $\rho_{n}^{\prime} \circ q^{\prime}=d \circ r$. This implies that $\rho_{n}^{\prime} \circ q^{\prime} \circ i$ is a coboundary with respect to the cohomology $H^{n+1}(\mathfrak{A} ; \operatorname{ker} q)$. The cohomology class $\left[q^{\prime} \circ i\right]$ lies in the kernel of

$$
\rho_{n}^{\prime n+1}: H^{n+1}\left(\Re(n-1)\left(W_{n}^{\prime}\right) ; \underline{\operatorname{ker}} q\right) \rightarrow H^{n+1}(\mathfrak{A} ; \underline{\operatorname{ker}} q)
$$

and in the kernel of the edge of homomorphism

$$
H^{n+1}\left(\mathfrak{M}(n-1)\left(W_{n}^{\prime}\right) ; \underline{\operatorname{ker}} q\right) \rightarrow \operatorname{Hom}\left(\underline{\operatorname{ker}} q, H^{n+1}\left(\mathfrak{M}(n-1)\left(W_{n}^{\prime}\right)\right)\right) .
$$

We again study the relation between the corresponding spectral sequences and we find that the only common element of the two kernels is 0 . This proves the claim above and the existence of $q^{\prime \prime}$.

We extend $q^{\prime \prime}$ to a morphism $\hat{q}: Q \rightarrow \mathfrak{M}(n-1)\left(W_{n}^{\prime}\right)^{n}$. The morphisms $q^{\prime}-d \circ \hat{q}$ and $r-\rho_{n}^{\prime} \circ \hat{q}$ are 0 when restricted to ker $q$. Therefore, they factor through $q$ via $d^{\prime \prime}: W_{n}^{\prime \prime} \rightarrow \underline{Z}^{n+1}\left(\mathfrak{M}(n-1)\left(W_{n}^{\prime}\right)\right)$ and $\rho_{n}^{\prime \prime}: \bar{W}_{n}^{\prime \prime} \rightarrow \mathfrak{U}^{n}$ respectively. Again, we consider the elementary extension of $\mathfrak{M}(n-1)\left(W_{n}^{\prime}\right)$ with respect to $d^{\prime \prime}$

$$
\mathfrak{M}(n) \equiv \mathfrak{M}(n-1)\left(W_{n}^{\prime}\right)\left(W_{n}^{\prime \prime}\right)
$$

and extend $\rho_{n}^{\prime}: \mathfrak{M}(n-1)\left(W_{n}^{\prime}\right) \rightarrow \mathfrak{A}$ and $\rho_{n}^{\prime \prime}$ to a morphism $\rho_{n}: \mathfrak{M}(n) \rightarrow \mathfrak{A}$.

The minimality of $\mathfrak{M}(n)$ can be seen as follows. We want to prove that the differential of $\mathfrak{M}(n)$, when restricted on $\bigcap_{H^{\prime} \supset H} \operatorname{ker} \mathfrak{M}(n)\left(\hat{e}_{H, H^{\prime}}\right) \subseteq \mathfrak{M}(n)(G / H)$, is decomposable for every $H \subseteq G$. Since $d$ restricted on $\cap_{H^{\prime} \supset H}$ ker $\mathfrak{M}(n-1)\left(\hat{e}_{H, H^{\prime}}\right)$ is decomposable by assumption, we have to check the differential on $\bigcap_{H^{\prime} \supset H} \operatorname{ker} W_{n, i}^{\prime}\left(\hat{e}_{H, H^{\prime}}\right)$ and on $\bigcap_{H^{\prime} \supset H} \operatorname{ker} W_{n, i}^{\prime \prime}\left(\hat{e}_{H, H^{\prime}}\right)$ for every $i \geqslant 0$. We start with $i=0$. We first note that if $M$ is in $\operatorname{Vec}_{G}^{*}$ and $I$ is its injective envelope then

$$
\bigcap_{H^{\prime} \supset H} \operatorname{ker} M\left(\hat{e}_{H, H^{\prime}}\right) \cong \bigcap_{H^{\prime} \supset H} \operatorname{ker} I\left(\hat{e}_{H ; H^{\prime}}\right)
$$

for all $H \subseteq G$ by our construction of injective envelopes. In our case

$$
\bigcap_{H^{\prime} \supset H} \operatorname{ker} W_{n}^{\prime}\left(\hat{e}_{H, H^{\prime}}\right) \cong \bigcap_{H^{\prime} \supset H} \operatorname{ker} W_{n, 0}^{\prime}\left(\hat{e}_{H, H^{\prime}}\right) ;
$$

the same is true for $W_{n}^{\prime \prime}$. The differential $d^{\prime}$ on $W_{n}^{\prime}$ is defined as $d \circ q^{\prime}$, where $d$ is the differential of $\mathfrak{M}(n-1)$. Therefore it is decomposable when restricted to (7.2). The differential $d^{\prime \prime}$ on $W_{n}^{\prime \prime}$ is defined as $q^{\prime}-d \circ \hat{q}$, where $d$ is the differential of $\mathfrak{M}(n-1)$. We only need to prove that $q^{\prime}\left(\bigcap_{H^{\prime} \supset H} \operatorname{ker} W_{n}^{\prime \prime}\left(\hat{e}_{H, H^{\prime}}\right)\right)$ is decomposable. In fact, the cocycle $q^{\prime}: Q \rightarrow \underline{Z}^{n+1}\left(\mathfrak{M}(n-1)\left(W_{n}^{\prime}\right)\right)$ can be chosen to take values in the decomposable part of $\underline{Z}^{n+1}\left(\mathfrak{M}(n-1)\left(W_{n}^{\prime}\right)\right)$.

In order to explain this, we first recall some facts about free DGA's. If $\mathscr{Q}$ is a free DGA, it can be written $\mathscr{Q}=\Re \otimes \mathbf{Q}(V, d V)$, where $\Re$ is a minimal DGA and 
$\mathbf{Q}(V, d V)$ is a free acyclic DGA generated by the graded vector space $V \oplus d V$ as an algebra and such that $d(V)=d V$. If $\Re=\Re(n)$, i.e., $\Re$ is generated by elements of degree less than or equal to $n$, then $Z^{n+1}(\mathbb{Q}) \cap \mathbb{Q}^{+} \wedge \mathbb{Q}^{+} \rightarrow H^{n+1}(\mathbb{Q})$ is surjective (every cohomology class can be represented by a decomposable cocycle). This is the case for each $\mathfrak{M}(n-1)\left(W_{n}^{\prime}\right)(G / H), H \subseteq G$, i.e.,

$$
\begin{gathered}
\underline{Z}^{n+1}\left(\mathfrak{M}(n-1)\left(W_{n}^{\prime}\right)\right) \cap \mathfrak{M}(n-1)\left(W_{n}^{\prime}\right)^{+} \wedge \mathfrak{M}(n-1)\left(W_{n}^{\prime}\right)^{+} \\
\rightarrow \underline{H}^{n+1}\left(\mathfrak{M}(n-1)\left(W_{n}^{\prime}\right)\right)
\end{gathered}
$$

is an epimorphism. Therefore $i \circ q: Q \rightarrow \underline{H}^{n+1}\left(\mathfrak{M}(n-1)\left(W_{n}^{\prime}\right)\right)$ has a lifting with respect to this epimorphism. Call the lifting $q^{\prime}$.

For $i>0$, similar arguments apply. This completes the proof of the minimality of $\mathfrak{M}(n)$.

In order to prove that

$$
\rho_{n}^{n}: \underline{H}^{n}(\mathfrak{M}(n)) \rightarrow \underline{H}^{n}(\mathfrak{U})
$$

is an isomorphism and that

$$
\rho_{n}^{n+1}: \underline{H}^{n+1}(\mathfrak{M}(n)) \rightarrow H^{n+1}(\mathfrak{U})
$$

is a monomorphism, we study the above construction for each $G / H, H \subseteq G$, separately. This presents no difficulty and we will omit the proof.

Proof of Corollary 5.11. Let $\mathfrak{M}$ be a minimal system of DGA's for $G$. According to the constructions of the proof of Theorem 5.3 there is a sequence

$$
\mathfrak{M}(1) \subseteq \cdots \subseteq \mathfrak{M}(n-1) \subseteq \mathfrak{M}(n) \subseteq \cdots
$$

of minimal systems of DGA's and morphisms $\rho_{n}: \mathfrak{M}(n) \rightarrow \mathfrak{A}$ with the properties (7.3) and (7.4). Consider the union $\mathfrak{M}^{\prime}=\cup_{n \geqslant 0} \mathfrak{M}(n)$ and the induced morphism $\rho$ : $\mathfrak{M}^{\prime} \rightarrow \mathfrak{M}$. Since $\rho$ is a cohomology isomorphism, it is an isomorphism. Moreover,

$$
0 \rightarrow \underline{\operatorname{coker}} \rho_{n-1}^{n} \rightarrow W_{n} \rightarrow \underline{\operatorname{ker}} \rho_{n}^{n+1} \rightarrow 0
$$

by construction. But this is equal to $\underline{H}^{n}(\mathfrak{M}(n), \mathfrak{M}(n-1))$ as we can see from the long exact sequence

$$
\begin{aligned}
\cdots & \rightarrow \underline{H}^{n}(\mathfrak{M}(n)) \rightarrow \underline{H}^{n}(\mathfrak{M}(n), \mathfrak{M}(n-1)) \rightarrow \underline{H}^{n+1}(\mathfrak{M}(n-1)) \\
& \rightarrow \underline{H}^{n+1}(\mathfrak{M}(n)) \rightarrow \cdots .
\end{aligned}
$$

For the proof of Propositions 5.4 and 5.5 we use ideas analogous to the ones of the nonequivariant case and the techniques of the construction used to prove Theorem 5.3. We content ourselves with an outline of the proof of 5.5: Let $\mathfrak{M}_{1}$ and $\mathfrak{M}_{2}$ be two minimal systems of DGA's for $G$ such that $\mathfrak{M}_{2}$ is an elementary extension of $\mathfrak{M}_{1}$ with respect to $d^{\prime}: W_{n} \rightarrow \underline{Z}^{n+1}\left(\mathfrak{M}_{1}\right)$. Let $f, g: \mathfrak{M}_{2} \rightarrow \mathfrak{A}$ be two morphisms and $H_{1}: \mathfrak{M}_{1} \rightarrow \mathfrak{U}(t, d t)$ a homotopy between $f \mid \mathfrak{M}_{1}$ and $g \mid \mathfrak{M}_{1}$. Then $H_{1}$ 
can be extended to a homotopy $\mathrm{H}_{2}$ between $f$ and $g$ if and only if the morphism $\sigma_{n}$ : $W_{n} \rightarrow \underline{Z}^{n+1}(\mathfrak{A})$ defined by

$$
\sigma_{n}=f-g+(-1)^{n} \sum_{m=0}^{M} \frac{1}{m+1} k_{m}
$$

is a coboundary; i.e., it factors through $d$ via some $\sigma_{n}^{\prime}: W_{n} \rightarrow \mathfrak{A}^{n} ; k_{m}: W_{n} \rightarrow \mathfrak{A}^{n}$ is a morphism such that

$$
H_{1} \circ d^{\prime}=\sum_{m=0}^{M} h_{m} t^{m}+\sum_{m=0}^{M} k_{m} t^{m} d t .
$$

Then the homotopy $\mathrm{H}_{2}$ is defined on $W_{n}$ by

$$
H_{2}=f+(-1)^{n} \sum_{m=0}^{M} \frac{1}{m+1} k_{m} t^{m+1}-d \circ \sigma^{\prime}
$$

and, by the techniques of 5.10 , it can be pxtended over $\mathfrak{M}_{2}$.

We shall use these facts to prove Proposition 5.5 as follows. Suppose inductively that there is $f_{n-1}^{\prime}: \mathfrak{M}(n-1) \rightarrow \mathfrak{A}$ such that the diagram

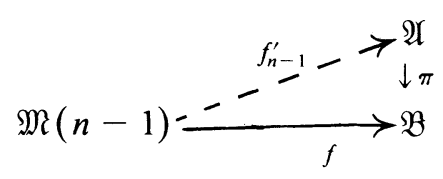

commutes up to homotopy. We want to extend it over $\mathfrak{M}(n)$, which is an elementary extension of $\mathfrak{M}(n-1)$ with respect to $d^{\prime}: W_{n} \rightarrow Z^{n+1}(\mathfrak{M}(n-1))$. The morphism

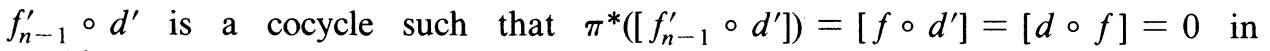
$H^{n+1}\left(\mathfrak{B}, W_{n}\right)$. Since $\pi^{*}$ is an isomorphism, $\left[f_{n-1}^{\prime} \circ d^{\prime}\right]=0$, i.e., $f_{n-1}^{\prime} \circ d^{\prime}$ factors throught the differential of $\mathfrak{A}$ via some $\tilde{f}_{n}: W_{n} \rightarrow \mathfrak{A}^{n+1}$. The obstruction to a homotopy between $f \mid \mathfrak{M}(n)$ and $\pi \circ f_{n}^{\prime}$ is $\left[\sigma_{n}\right] \in H^{n+1}\left(\mathfrak{B}, W_{n}\right)$, where

$$
\sigma_{n}=f \mid \mathfrak{M}(n)-\pi \circ \tilde{f}_{n}+(-1)^{n} \sum_{m=0}^{M} \frac{1}{m+1} k_{m} .
$$

Let $\left[\tilde{\sigma}_{n}\right] \in H^{n+1}\left(\mathfrak{A}, W_{n}\right)$ be such that $\pi^{*}\left[\tilde{\sigma}_{n}\right]=\left[\sigma_{n}\right]$. Then $\pi \circ \tilde{\sigma}_{n}-\sigma_{n}=d \circ \sigma_{n}^{\prime}$ (the difference is a coboundary). We correct $\tilde{f}_{n}$ to $f_{n}^{\prime}=\tilde{f}_{n}-\tilde{\sigma}_{n}$ and extend this over $\mathfrak{M}(n)$. The obstruction to a homotopy between $\pi \circ f_{n}^{\prime}$ and $f \mid \mathfrak{M}(n)$ now vanishes.

\section{Deferred proofs from $\$ 6$.}

Proof OF Lemma 6.3. First of all we have to determine the concept of equivariant transgression. Since

$$
F^{H}=K(M(G / H), n) \rightarrow E^{H} \rightarrow X^{H}
$$

is a transgressive fibration and since the transgression is functorial, there is a morphism $\tau: \underline{H}^{n}(F ; \mathbf{Q}) \rightarrow \underline{H}^{n+1}(X ; \mathbf{Q})$ such that the corresponding part of the following diagram commutes and, in particular, $p^{*} \circ \tau=0$. 


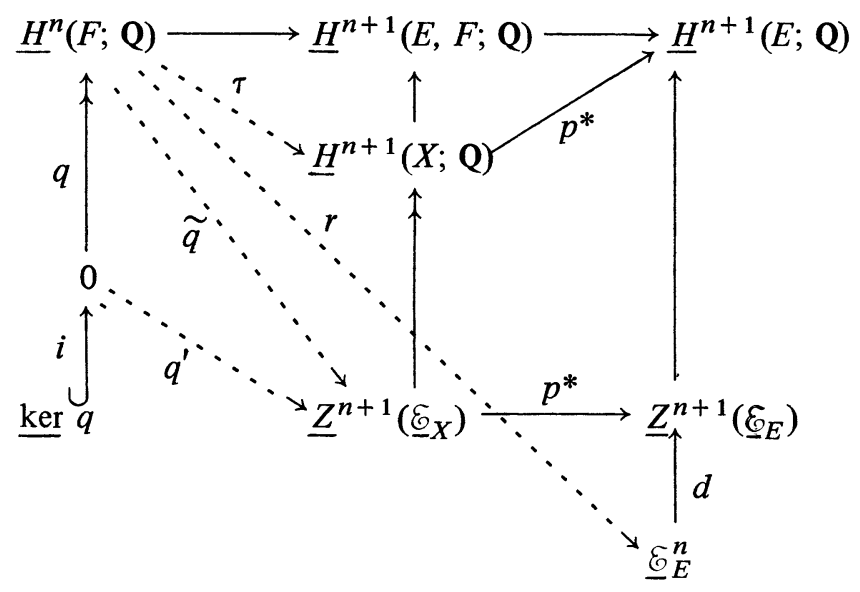

Let $q: Q \rightarrow \underline{H}^{n}(F ; \mathbf{Q})$ be an epimorphism, where $Q$ is projective in $\operatorname{Vec}_{G}^{*}$. Then there is a lifting $q^{\prime}$ of $\tau \circ q$. Moreover, $q^{\prime} \circ i$ : $\operatorname{ker} q \rightarrow \underline{Z}^{n+1}\left(\underline{\mathcal{E}}_{X}\right)$ is a morphism into the exact $P L$ forms $\underline{B}^{n+1}\left(\underline{\mathscr{E}}_{X}\right) \subseteq \underline{Z}^{n+1}\left(\underline{\mathscr{\varepsilon}}_{X}\right)$. We have the same situation as in the second diagram of the proof of 5.3. If we use the same method, we find morphisms $\tilde{q}$ and $r$ such that the entire diagram commutes.

Since $\rho: \mathfrak{A} \rightarrow \underline{\mathcal{E}}_{X}$ induces an isomorphism on cohomology and, in particular,

$$
\rho^{*}: H^{n+1}\left(\mathfrak{A} ; \underline{H}^{n}(F, \mathbf{Q})\right) \stackrel{\cong}{\rightarrow} H^{n+1}\left(\underline{\mathcal{E}}_{X} ; \underline{H}^{n}(F ; \mathbf{Q})\right) \text {, }
$$

there is a morphism $\sigma: \underline{H}^{n}(F ; \mathbf{Q}) \rightarrow \underline{Z}^{n+1}(\mathfrak{A})$ such that $\rho^{*}[\sigma]=[\tilde{q}]$; then $\rho \circ \sigma=\tilde{q}$ after possible correction of $\tilde{q}$ and $r$ by a coboundary.

We consider the elementary extension $\tilde{\mathfrak{A}}=\mathfrak{A}\left(\underline{H}^{n}(F ; \mathbf{Q})\right)$, where $d: \underline{H}^{n}(F ; \mathbf{Q}) \rightarrow$ $\underline{Z}^{n+1}(\mathfrak{A})$ is equal to $\sigma$ and we define a morphism $\tilde{\rho}: \tilde{\mathfrak{U}} \rightarrow \underline{\mathcal{E}}_{E}$ such that $\tilde{\rho} \mid \mathfrak{A}=\rho$ and $\tilde{\rho} \mid H^{n}(F ; \mathbf{Q})=r$.

In order to prove that $\tilde{\rho}$ induces an isomorphism on cohomology, we consider the construction for each $G / H, H \subseteq G$, and apply the (nonequivariant) arguments of [3].

Proof of Lemma 6.4. The long exact sequence (4.8) implies $\underline{H}^{i}(\tilde{\mathfrak{A}}, \mathfrak{A})=0, i<n$ and $\underline{H}^{n}(\tilde{\mathfrak{A}}, \mathfrak{A}) \cong W_{n}$. On the other hand, the spectral sequence (4.7) yields $H^{n}\left(\tilde{\mathfrak{A}}, \mathfrak{A} ; W_{n}\right) \cong \operatorname{Hom}\left(W_{n}, W_{n}\right)$. We construct a $G$-fibration $F=K\left(W_{n}^{*}, n\right) \rightarrow E \rightarrow X$ the equivariant $k$-invariant of which is the image of [id] $\in H^{n}\left(\tilde{\mathfrak{A}}, \mathfrak{A} ; W_{n}\right)$ under

$$
\delta^{*}: H^{n}\left(\tilde{\mathfrak{U}}, \mathfrak{A} ; W_{n}\right) \rightarrow H^{n+1}\left(\mathfrak{A} ; W_{n}\right) \cong H_{G}^{n+1}\left(X ; W_{n}^{*}\right) .
$$

Since (id, $-\tilde{d}): W_{n} \rightarrow \tilde{\mathfrak{A}} \times \mathfrak{A}$ represents the cohomology class [id], the morphism $-\tilde{d}$ : $W_{n} \rightarrow \mathfrak{A}^{n+1}$ represents the $k$-invariant.

In order to show that $\tilde{\mathfrak{A}}$ is geometric for $E$ we shall construct a system of DGA's $\hat{\mathfrak{A}}$ which is geometric for $E$ (according to the previous lemma) and show that $\tilde{\mathfrak{U}}$ and $\hat{\mathfrak{U}}$ are isomorphic. $\hat{\mathfrak{A}}$ is an elementary extension of $\mathfrak{A}$ with respect to some morphism $\hat{d}$ : $\underline{H}^{n}(F ; \mathbf{Q}) \rightarrow \underline{Z}^{n+1}(\mathfrak{A})$ such that $\rho \circ \hat{d}$ induces the transgression $\tau: \underline{H}^{n}(F ; \mathbf{Q}) \rightarrow$ $\underline{H}^{n+1}(X ; \mathbf{Q})$. Since $\underline{H}^{n}(F ; \mathbf{Q})=\underline{H}^{n}\left(K\left(W_{n}^{*}, n\right) ; \mathbf{Q}\right)=W_{n}$ (this follows from the nonequivariant case) we have

$$
H^{n}\left(\hat{\mathfrak{A}}, \mathfrak{U} ; W_{n}\right) \cong \operatorname{Hom}\left(W_{n}, W_{n}\right) \cong \operatorname{Hom}\left(W_{n}^{*}, W_{n}^{*}\right)=\operatorname{Hom}\left(\underline{\pi}_{n}(F), \underline{\pi}_{n}(F)\right) .
$$


According to our discussion in (2.5), the equivariant $k$-invariant of the above $G$-fibration is the image of [id] under

$$
\hat{\delta}^{*}: H^{n}\left(\hat{\mathfrak{U}}, \mathfrak{A} ; W_{n}\right) \cong \operatorname{Hom}\left(\underline{\pi}_{n}(F), \underline{\pi}_{n}(F)\right) \rightarrow H^{n+1}\left(\mathfrak{A}, W_{n}\right) \cong H_{G}^{n+1}\left(X ; \underline{\pi}_{n}(F)\right) ;
$$

therefore it can be represented by $-\hat{d}$.

The morphisms $\tilde{d}$ and $\hat{d}$ differ by a coboundary $\tilde{d}-\hat{d}=d \circ \sigma, \sigma: W_{n} \rightarrow \mathfrak{A}^{n}$, because they represent the same cohomology class.

We define $A: \tilde{\mathfrak{U}} \rightarrow \hat{\mathfrak{A}}$ by $\left.A\right|_{\mathfrak{A}}=\mathrm{id}$ and $\left.A\right|_{W_{n}}=\mathrm{id}+\sigma$. This is obviously an isomorphism.

Proof of Proposition 6.7. Our construction of the equivariant minimal model allows the transfer of the nonequivariant arguments to our case. We shall construct $f$ inductively as a map between the equivariant Postnikov decomposition of $X$ and $Y$. The other statements of the proposition are proved similarly.

Let $f_{n-1}: X_{n-1} \rightarrow Y_{n-1}$ be a $G$-map which induces $\tilde{f} \mid \mathfrak{A}(n-1): \mathfrak{R}(n-1) \rightarrow$ $\mathfrak{M}(n-1)$. The first obstruction to lifting $f_{n-1}$ to a $G$-map $h: X_{n} \rightarrow Y_{n}$ is the cohomology class $p_{X}^{*} f_{n-1}^{*}\left(k^{n+1}\right) \in H_{G}^{n+1}\left(X_{n-1} ; \underline{\pi}_{n}(Y)\right)$.

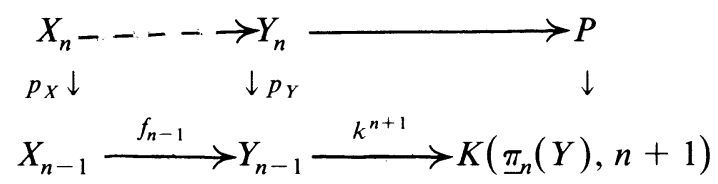

But $p_{X}^{*} f_{n-1}^{*}\left(k^{n+1}\right)=\tilde{f}^{*} p_{Y}^{*}\left(k^{n+1}\right)=\tilde{f}^{*}(0)=0$ in $H^{n+1}\left(\Re(n) ; \pi_{n}(Y)\right)$.

Let $\tilde{h}: \mathfrak{R}(n) \rightarrow \mathfrak{M}(n)$ be a morphism induced by $h$ such that $\tilde{h} \mid \mathfrak{R}(n-1)=$ $\tilde{f} \mid \mathfrak{R}(n-1)$. The morphisms $\tilde{h}$ and $\tilde{f}$ need not be homotopic on $\mathfrak{R}(n)$. We consider the difference

$$
\Delta: V_{n} \rightarrow \mathfrak{M}(n), \quad \Delta=\tilde{h}\left|V_{n}-\tilde{f}\right| V_{n},
$$

where $\mathfrak{N}(n)=\mathfrak{R}(n-1)\left(V_{n}\right)$, and the cohomology class it represents. According to the classification theorem (2.4), we can change the lifting $h$ by $[\Delta]$ to a lifting $f_{n}$ : $X_{n} \rightarrow Y_{n}$. Then the new $\Delta$ is a coboundary and thus, by the discussion in the proof of 5.5, $\tilde{f}_{n}$ and $\tilde{f} \mid \mathfrak{R}(n)$ are homotopic.

\section{REFERENCES}

1. G. E. Bredon, Equivariant cohomology theories, Lecture Notes in Math., vol. 34, Springer-Verlag, Berlin, Heidelberg and New York, 1967.

2. A. K. Bousfield and V. K. A. M. Gugenheim, On PL de Rham theory and rational homotopy type, Mem. Amer. Math. Soc., no. 179 (1976).

3. P. Deligne, P. Griffiths, J. Morgan and D. Sullivan, Real homotopy theory of Kähler manifolds, Invent. Math. 29 (1975), 245-274.

4. S. Mac Lane, Homology, Die Grundlehren der math. Wissenschaften, Bd. 114, Springer-Verlag, Berlin, 1963.

5. G. V. Triantafillou, Äquivariante rationale Homotopietheorie, Bonner Math. Schriften Nr. 110, 1978.

6. H. Whitney, Geometric integration theory, Princeton Univ. Press, Princeton, N. J., 1957.

School of Mathematics, Institute for Advanced Study, Princeton, New Jersey 08540

Department of Mathematics, University of Chicago, Chicago, Illinois 60637

Current address: Department of Mathematics, University of Minnesota, Minneapolis, Minnesota 55455 